\title{
Surface defects on E-string from 5-brane webs
}

\author{
Sung-Soo Kim, ${ }^{a}$ Yuji Sugimoto ${ }^{b, c}$ and Futoshi Yagi ${ }^{d}$ \\ ${ }^{a}$ School of Physics, University of Electronic Science and Technology of China, \\ No. 2006, Xiyuan Ave, West Hi-Tech Zone, Chengdu, Sichuan 611731, China \\ ${ }^{b}$ Interdisciplinary Center for Theoretical Study, University of Science and Technology of China, \\ 96 Jinzai Road, Hefei, Anhui 230026, China \\ ${ }^{c}$ Peng Huanwu Center for Fundamental Theory, \\ 96 Jinzai Road, Hefei, Anhui 230026, China \\ ${ }^{d}$ School of Mathematics, Southwest Jiaotong University, \\ West zone, High-tech district, Chengdu, Sichuan 611756, China \\ E-mail: sungsoo.kim@uestc.edu.cn, sugimoto@ustc.edu.cn, \\ futoshi_yagi@swjtu.edu.cn
}

ABSTRACT: We study 6d E-string theory with defects on a circle. Our basic strategy is to apply the geometric transition to the supersymmetric gauge theories. First, we calculate the partition functions of the $5 \mathrm{~d} \mathrm{SU}(3)_{0}$ gauge theory with 10 flavors, which is UV-dual to the $5 \mathrm{~d} \mathrm{Sp}(2)$ gauge theory with 10 flavors, based on two different 5-brane web diagrams, and check that two partition functions agree with each other. Then, by utilizing the geometric transition, we find the surface defect partition function for E-string on $\mathbb{R}^{4} \times T^{2}$. We also discuss that our result is consistent with the elliptic genus. Based on the result, we show how the global symmetry is broken by the defects, and discuss that the breaking pattern depends on where/how we insert the defects.

KEYwords: D-branes, Global Symmetries, String Duality, Supersymmetric Gauge Theory

ARXIV EPRINT: 2008.06428 


\section{Contents}

1 Introduction 1

2 5d SU(3) gauge theories, 5-brane webs, and UV duality 3

2.1 Partition function from Tao diagram 4

$\begin{array}{lll}2.2 & \text { 5-brane web with two O5-planes } & 7\end{array}$

3 Defect partition function of E-strings $\quad 10$

3.1 Usual Higgsing on 5-brane webs 11

3.2 Defect Higgsing on 5-brane webs 13

$\begin{array}{ll}\text { 3.3 Defect Higgsing in elliptic genus and global symmetry } & 17\end{array}$

4 Global symmetry $\quad 21$

5 Conclusion $\quad 22$

A Conventions and notations $\quad 24$

A.1 Topological vertex 24

A.2 Theta function $\quad 25$

A.3 Plethystic exponential 26

B Kähler parameters of the Tao diagram 26

C $(p, q)$ 5-brane web with O5-planes and topological vertex 28

C.1 Topological vertex formalism with O5-planes 28

$\begin{array}{ll}\text { C.2 The partition function of } \mathrm{SU}(N+1)_{0}+(2 N+6) \mathbf{F} & 29\end{array}$

$\begin{array}{ll}\text { D Flavor decoupling limit } & 29\end{array}$

E Defects on pure SU(2) theories with different discrete theta angles 32

\section{Introduction}

Study of surface defects $[1,2]$ provides us with a tool for uncovering novel structure of non-perturbative aspects as well as enriching interplay between theories of codimension 2 [3-9]. A way to see how the defects affect physical systems is to calculate the partition functions. It is known that partition functions for some class of $5 \mathrm{~d} \mathcal{N}=1$ supersymmetric gauge theories compactified on a circle are equivalent to topological string amplitudes on corresponding non-compact toric Calabi-Yau manifolds under suitable parameter correspondence, which is known as geometric engineering [10-13]. Based on the equivalence 
between toric Calabi-Yau manifold and 5-brane web [14], we can translate the geometry into brane set up and vice versa.

In the presence of the defects, the partition function can be computed by implementing $5 \mathrm{~d}$ system with the defects on a Type IIB 5-brane web, where the defects are realized as (perpendicular) D3-branes inserted on the $(p, q)$-web plane for supersymmetric gauge theories. Such defect insertion is captured as a particular choice of Kähler parameters in topological string amplitudes, which corresponds to open topological string amplitudes [15, $16]$, which is known as geometric transition [17, 18].

The procedure of obtaining the defect partition functions can be viewed as a generalization of Higgsing procedure from 5-brane webs. Depending how to choose or tune the Kähler parameters, one sees usual Higgsing or a system with defect. More precisely, to obtain the defect partition function, we need to tune Kähler parameters in a way that not only it reduces the rank of gauge group but also yields the open topological string partition function up to Coulomb branch independent overall factors such as MacMahon function or the extra factors. Geometric transition when the number of inserted D3 defects is zero, reduces to usual Higgsing. In this regard, it is a generalization of Higgsing procedure, and we refer to it as defect Higgsings. Using the defect Higgsing, the 5d defect partition functions have been computed in a straightforward manner.

Many $6 \mathrm{~d}$ theories on a circle are also realized on 5-brane webs, one can hence also apply the defect Higgsing to such KK theories. One example is M-string theory with surface defects inserted [19], where M-string is realized as a periodic $(p, q)$ 5-brane webs where the NS5-branes are identified. This gives rise to a web on a cylinder where the circular direction corresponds to the compactification direction of $6 \mathrm{~d}$ theories. [20, 21].

As another example of defect Higgsing, in this paper, we study 6d E-string theory with surface defects. E-string theory on a circle of radius $R$ is realized as $\mathrm{SU}(2)$ gauge theory with 8 hypermultiplets in the fundamental representation (flavors). There are two different 5-brane webs for the $\mathrm{SU}(2)$ gauge theory. One is of a spirally periodic shape whose period is identified as $R^{-1}$, which is called Tao diagram [22]. The other is of two O5-planes with their distance $R^{-1}$. Since an orientifold plane is used to realize Sp or SO gauge group in general, we refer to the former 5-brane web as web diagram for $\mathrm{SU}(2)$ gauge theory, while the latter as web diagram for $\mathrm{Sp}(1)$ gauge theory in this paper even though $\mathrm{SU}(2)$ and $\mathrm{Sp}(1)$ are identical. Partition function can be computed based on 5-brane webs via topological vertex [23]. In particular, topological vertex formalism in the presence of O5-planes is also developed in [24] and both topological string partition functions agree with the elliptic genus of $6 \mathrm{~d}$ E-string theory.

Our strategy of computing the E-string partition function with defects is to first consider $5 \mathrm{~d} \mathrm{SU}(3)_{0}$ gauge theory with 10 flavors and then apply the defect Higgsing. The $5 \mathrm{~d}$ $\mathrm{SU}(3)_{0}$ gauge theory with 10 flavors is UV-dual to the $5 \mathrm{~d} \mathrm{Sp}(2)$ theory with 10 flavors, in the sense that their UV completion is the same [25, 26]. The corresponding $6 \mathrm{~d}$ theory description is given by $6 \mathrm{~d} \mathrm{Sp}(1)$ gauge theory with 10 flavors and a tensor multiplet. Together with the brane configurations, these dual descriptions allow one to compute the partition function in several different ways. For instance, the ADHM-like method [27], the elliptic genus [28], and the topological vertex method [29]. We compute the defect partition 
function by using the topological vertex method and compare it with the elliptic genus for E-string with defects which is obtained from $6 \mathrm{~d} \mathrm{Sp}(1)$ gauge theory with 10 flavors.

Analogous to the case with the $5 \mathrm{~d} \mathrm{SU}(2)$ gauge theory, the $5 \mathrm{~d} \mathrm{SU}(3)_{0}$ gauge theory with 10 flavors has two different 5-brane configurations: one is without O5-plane and the other is with two O5-planes. The 5-brane configuration without O5-plane is Tao diagram [22]. This Tao diagram can be obtained from the 5 -brane web diagram with two $\mathrm{O}^{-}$-planes, which is T-dual of the type IIA brane setup with an $\mathrm{O}^{-}$-plane [30, 31] for $6 \mathrm{~d} \mathrm{Sp}(1)$ gauge theory with 10 flavors and a tensor multiplet. By resolving both $\mathrm{O}^{-}$-planes into two different 7-branes [32], respectively, we obtain the diagram for the $5 \mathrm{~d} \mathrm{SU}(3)_{0}$ gauge theory, which can be deformed to be a Tao diagram. On the other hand, if we resolve only one $\mathrm{O}^{-}{ }^{-}$ plane, we obtain the diagram for the $5 \mathrm{~d} \operatorname{Sp}(2)$ gauge theory, which explains the UV-duality between the $\mathrm{SU}(3)$ gauge theory and the $\mathrm{Sp}(2)$ gauge theory [25, 26, 29]. The 5-brane configuration with two O5-planes is again the T-dual of the type IIA brane setup but with O6-plane instead of O8-plane. As abuse of notation, we refer to the former web diagram without O5-planes as the web diagram for $\mathrm{SU}(3)$ gauge theory, while the latter diagram with two O5-planes as the web diagram for $\operatorname{Sp}(2)$ gauge theory in this paper, based on the knowledge that orientifold planes are used to realize Sp or SO gauge group in general. ${ }^{1}$

The organization of the paper is as follows. In section 2 , we review the partition function for $5 \mathrm{~d} \mathrm{SU}(3)_{0}$ gauge theory with 10 flavors from two different 5-branes setups: one with a 5-brane web without O5-planes and the other with two O5-planes. In section 3, using $5 \mathrm{~d}$ 5-brane configurations for the $\mathrm{SU}(3)_{0}$ gauge theory with 10 flavors, we perform defect Higgsing to yields $5 \mathrm{~d} \mathrm{SU}(2)$ gauge theory with 8 flavors. For comparison, we implement the defect Higgsing to the elliptic genus partition function of E-string theory to the agreement. In section 4, we discuss some issues of global symmetry in the presence of defects. We then conclude and discuss unbroken global symmetry and possible generalizations. In appendix, we discuss our conventions, decoupling limit to get 5 d theories from KK theories, and defects on pure $\mathrm{SU}(2)$ theories with different discrete theta angles.

\section{$25 \mathrm{~d} \mathrm{SU}(3)$ gauge theories, 5-brane webs, and UV duality}

In this section, we consider the $5 \mathrm{~d} \mathcal{N}=1 \mathrm{SU}(3)_{\kappa}$ gauge theories from the perspective of 5-brane webs in Type IIB string theory. In particular, we discuss how to obtain the BPS partition function of the $\mathrm{SU}(3)_{0}$ gauge theory with 10 flavors of the Chern-Simons level $\kappa=0$. The computation is performed based on two different 5 -brane web diagrams. One is a 5-brane web diagram without O5-planes, which is Tao diagram introduced in [22]. Though it is spirally periodic, one can apply the topological vertex method to compute the unrefined Nekrasov partition function. The other is a 5-brane web with two O5-planes. It is also possible to implement the topological vertex method to the 5-brane configurations

\footnotetext{
${ }^{1}$ Rigorously speaking, it would be more proper to understand that we obtain $5 \mathrm{~d}$ SU(3) gauge theory or $5 \mathrm{~d} \mathrm{Sp}(2)$ gauge theory depending on the parameter region of the Wilson lines introduced to the $6 \mathrm{~d}$ superconformal theory realized at the UV fixed point as well as the compactification radius. It indicates that both gauge theories can be realized in either of the two 5-brane web configurations. However, we use this notation just for simplicity.
} 
with O5-plane(s) with special deformation of web diagrams and careful Young diagram assignments near O5-planes [24].

The partition function for $5 \mathrm{~d} \mathrm{SU}(2)$ with 8 flavors has been already computed explicitly $[22,24,33]$. The resulting partition functions based on a Tao diagram [22] and a 5-brane with two O5-planes [24] look quite different, however, they agree up to unphysical factors, called extra factor, which do not depend on the Coulomb branch parameters. As for the $5 \mathrm{~d} \mathcal{N}=1 \mathrm{SU}(3)_{0}$ gauge theory with 10 flavors, the topological string partition function based on a Tao diagram has been already computed [29], but it has not been computed explicitly based on a 5-brane web with two O5-planes yet. In section 2.1, we first review the computation with the topological vertex based on the Tao diagram. Then, in section 2.2, we compute the partition function based on the 5-brane web with two O5planes. We refer to the former partition function as the partition function for $5 \mathrm{~d} \mathcal{N}=1$ $\mathrm{SU}(3)_{0}$ gauge theory with 10 flavors, while the latter one as the partition function for $5 \mathrm{~d}$ $\mathcal{N}=1 \mathrm{Sp}(2)$ gauge theory with 10 flavors. We will see agreement between them by expressing the latter partition function in terms of the parameters of the $\mathrm{SU}(3)$ gauge theory. The detailed computations and some notations are summarized in appendix A.

\subsection{Partition function from Tao diagram}

We first briefly review the computation of the partition function for $5 \mathrm{~d} \mathcal{N}=1 \mathrm{SU}(3)_{0}$ gauge theory with 10 flavors based on a 5-brane web [29]. A 5-brane configuration for such 5d marginal theories is called Tao web diagram [22], which is of a spiral shape with a periodic structure whose periodicity is given by the instanton factor squared. A Tao web diagram for $5 \mathrm{~d} \mathcal{N}=1 \mathrm{SU}(3)_{0}$ gauge theory with 10 flavors is depicted in figure 1(a). Though this 5-brane configuration, in principle, has infinitely many Kähler parameters, they are not independent due to the periodic structure of the web diagram, and only 13 parameters corresponding to the two Coulomb moduli parameters, ten mass parameters, and one instanton factor are independent. For computations, only half of the diagram suffices due to the symmetry of the web diagram. For instance, in order to compute figure 1(a), we only need to consider figure 1(b). This diagram in figure 1(b) can be further untangled with basic building blocks painted in different colors: middle strips, spiral strip 1, and spiral strip 2 as in figure 2. The assignment of the independent Kähler parameters $\left(Q_{i}\right)$ and Young diagrams $\left(\mu_{I}\right)$ are depicted in figure 2. The (unrefined) partition function is then obtained by gluing these building blocks with suitable edge factors.

The partition function for the $5 \mathrm{~d} \mathrm{SU}(3)_{0}$ gauge theory with 10 flavors then reads

$$
Z^{\mathrm{SU}(3)+10 \mathrm{~F}}=\sum_{\mu_{1,2,3}} Z_{\text {glue }}\left(Q_{b_{1}}, \mu_{1}\right) Z_{\text {glue }}\left(Q_{b_{2}}, \mu_{2}\right) Z_{\text {glue }}\left(Q_{b_{3}}, \mu_{3}\right) Z_{\text {half } 1} Z_{\text {half } 2} .
$$

Here, $Z_{\text {glue }}$ are the three edge factors for gluing, given by

$$
Z_{\text {glue }}(Q, \mu)=(-Q)^{|\mu|} q^{\frac{\left\|\mu||^{2}+|| \mu^{2}\right\|^{2}}{2}} \prod_{(i, j) \in \mu} \frac{1}{\left(1-q^{\mu_{i}+\mu_{j}^{t}-i-j+1}\right)^{2}},
$$

where $q=e^{-\beta \epsilon}$ is the parameter associated with the string coupling, defined with the selfdual $\Omega$-deformation parameter $\epsilon=\epsilon_{1}=-\epsilon_{2}$. $Z_{\text {half } 1}$ and $Z_{\text {half } 2}$ are the contributions of 
(a)

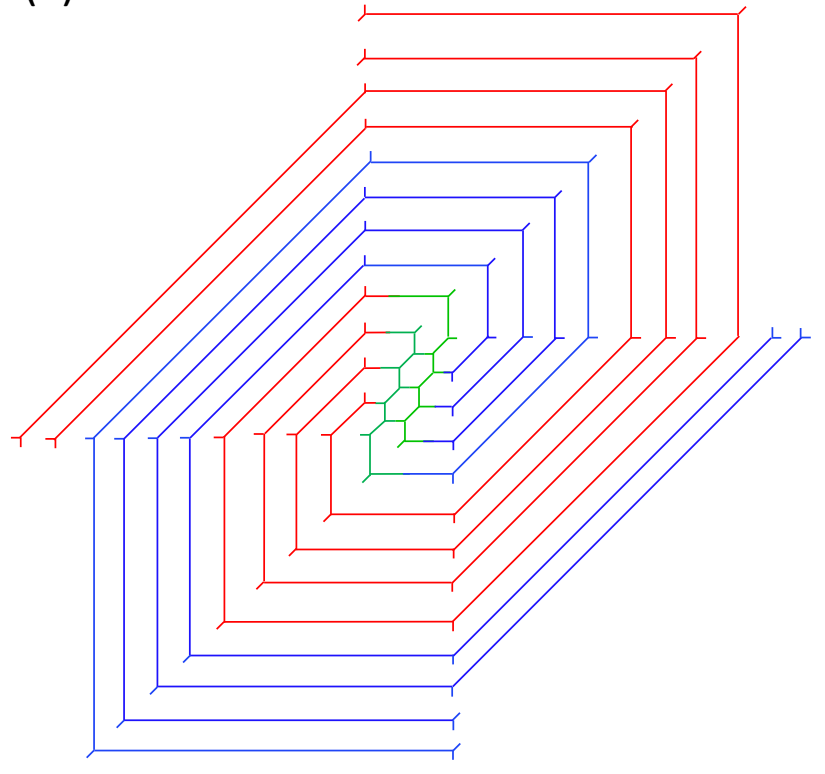

(b)

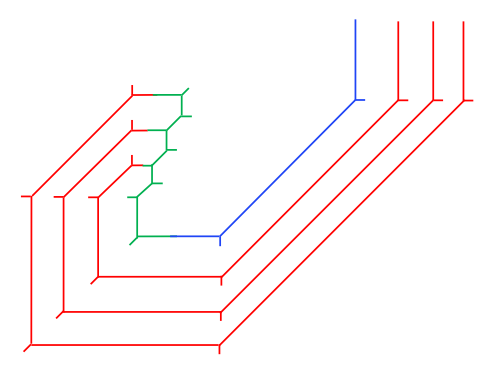

Figure 1. (a) The Tao diagram corresponding to the $5 \mathrm{~d} \mathcal{N}=1 \mathrm{SU}(3)$ gauge theory with 10 flavors whose spiral structure continues infinitely. (b) Its building block.

(a)

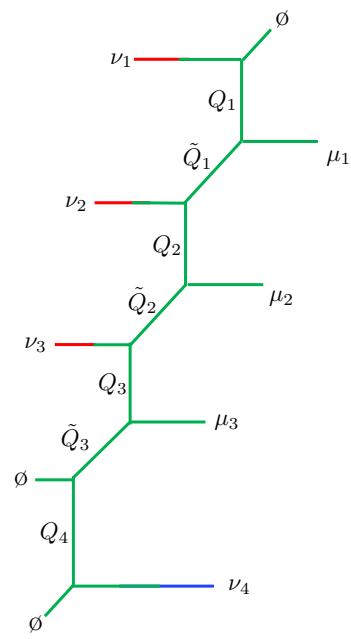

(b)

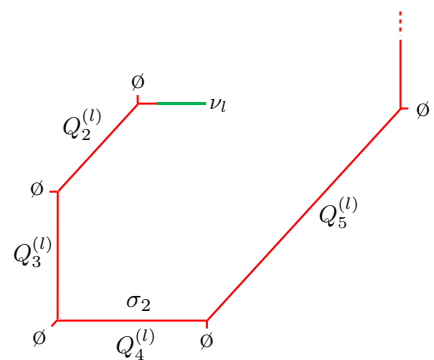

spiral strip 1 (c)

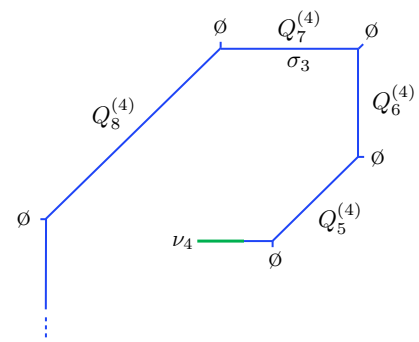

spiral strip 2

\section{middle strip}

Figure 2. The three kinds of building blocks of figure 1 (b). Here we call them (a) middle strip, (b) spiral strip 1, and (c) spiral strip 2. Greek letters $\nu_{l}(i=1,2,3)$ denote the Young diagrams. 
the half diagram in figure 1(b) and the other half, which are composed of three building blocks in figure 2 ,

$$
\begin{aligned}
Z_{\text {half } 1}= & \sum_{\nu_{1,2,3,4}} Z_{\text {middle }}\left(\nu_{1}, \nu_{2}, \nu_{3}, \varnothing, \mu_{1}, \mu_{2}, \mu_{3}, \nu_{4},\{\boldsymbol{Q}\},\{\tilde{\boldsymbol{Q}}\}\right) \\
& \times \prod_{l=1}^{3} Z_{\text {spiral } 1}\left(\left\{\boldsymbol{Q}^{(l)}\right\}, \nu_{l}\right) \times Z_{\text {spiral } 2}\left(\left\{\boldsymbol{Q}^{(4)}\right\}, \nu_{4}\right),
\end{aligned}
$$

where

$$
\begin{array}{rlrl}
\{\boldsymbol{Q}\} & =\left\{Q_{1}, Q_{2}, Q_{3}, Q_{4}\right\}, \quad\{\tilde{\boldsymbol{Q}}\} & =\left\{\tilde{Q}_{1}, \tilde{Q}_{2}, \tilde{Q}_{3}\right\} \\
\left\{\boldsymbol{Q}^{(k)}\right\} & =\left\{Q_{1}^{(k)}, Q_{2}^{(k)}, Q_{3}^{(k)}, \ldots\right\} \quad(k=1,2,3,4)
\end{array}
$$

Each of which takes the form ${ }^{2}$

$$
\begin{aligned}
Z_{\text {middle }}\left(\mu_{1}, \mu_{2}, \mu_{3}, \mu_{4}, \nu_{1}, \nu_{2}, \nu_{3}, \nu_{4},\{\boldsymbol{Q}, \tilde{\boldsymbol{Q}}\}\right) & \prod_{1 \leq i \leq j \leq 4} R_{\mu_{i} \nu_{j}}\left(Q_{j} \prod_{k=1}^{j-1} Q_{k} \tilde{Q}_{k}\right) \prod_{1 \leq i<j \leq 4} R_{\nu_{i} \mu_{j}}\left(\tilde{Q}_{i} \prod_{k=i+1}^{j-1} Q_{k} \tilde{Q}_{k}\right) \\
\prod_{\text {spiral } 1}\left(\{\boldsymbol{Q}\}, \nu_{l}\right)= & \sum_{\sigma_{2,3, \ldots}} R_{\mu_{i} \mu_{j}}\left(\prod_{k=1}^{j-1} Z_{\text {glue }}\left(Q_{1}, \nu_{l}\right) \frac{R_{\nu_{l} \phi}\left(Q_{2}\right) R_{\phi \sigma_{2}^{t}}\left(Q_{3}\right)}{R_{\nu_{l} \sigma_{2}^{t}}\left(Q_{2} Q_{3}\right)} R_{\nu_{i} \nu_{j}}\left(\prod_{k=i}^{j-1} Q_{k+1} \tilde{Q}_{k}\right)\right. \\
& \times \prod_{k=2}^{\infty} Z_{\text {glue }}\left(Q_{3 k-2}, \sigma_{k}\right) \frac{R_{\sigma_{k} \phi}\left(Q_{3 k-1}\right) R_{\phi \sigma_{k+1}^{t}}\left(Q_{3 k}\right)}{R_{\sigma_{k} \sigma_{k+1}^{t}}\left(Q_{3 k-1} Q_{3 k}\right)} \\
Z_{\text {spiral } 2}\left(\left\{\boldsymbol{Q}^{(4)}\right\}, \nu_{4}\right)= & \sum_{\sigma_{3,4, \ldots}} Z_{\text {glue }}\left(Q_{4}, \nu_{4}\right) \frac{R_{\nu_{4} \phi}\left(Q_{5}\right) R_{\phi \sigma_{3}^{t}}\left(Q_{6}\right)}{R_{\nu_{4} \sigma_{3}^{t}}\left(Q_{5} Q_{6}\right)} \\
& \times \prod_{k=3}^{\infty} Z_{\text {glue }}\left(Q_{3 k-2}, \sigma_{k}\right) \frac{R_{\sigma_{k} \phi}\left(Q_{3 k-1}\right) R_{\phi \sigma_{k+1}^{t}}}{R_{\sigma_{k} \sigma_{k+1}^{t}}\left(Q_{3 k-1} Q_{3 k}\right)}
\end{aligned}
$$

Here we define some quantities: $\nu_{i}$ are the Young diagrams along the horizontal lines. $R_{\mu \nu}(Q)$ is a function giving the contributions coming from the string wrapping on the internal line characterized by Kähler moduli $Q$ with Young diagrams $\mu$ and $\nu$,

$$
R_{\mu \nu}(Q)=\prod_{i, j=1}^{\infty}\left(1-Q q^{i+j-\mu_{i}-\nu_{j}^{t}-1}\right) .
$$

$Z_{\text {half2 }}$ can be obtained by suitable parameter replacement as the lower half (half1) and upper half (half2) of the diagram are symmetric.

After removing the extra factor, and using the proper flop transition (A.12) for the perturbative part, one finds that

$$
Z^{\mathrm{SU}(3)+10 \mathrm{~F}}=\mathrm{PE}\left[F_{0}(\{\boldsymbol{A}\},\{\boldsymbol{M}\}, q)+F_{1}(\{\boldsymbol{A}\},\{\boldsymbol{M}\}, q) \mathfrak{q}+F_{2}(\{\boldsymbol{A}\},\{\boldsymbol{M}\}, q) \mathfrak{q}^{2}+\mathcal{O}\left(\mathfrak{q}^{3}\right)\right],
$$

\footnotetext{
${ }^{2}(2.5 \mathrm{a})$ is slightly different from eq. (3.8) of [29], which has a typo in it. This typo in version 2 of [29] is corrected in $(2.5 \mathrm{a})$.
} 
where $\{\boldsymbol{A}\}=\left\{A_{1}, A_{2}, A_{3}\right\}$ and $\{\boldsymbol{M}\}=\left\{M_{1}, M_{2}, \ldots, M_{10}\right\}$ are Coulomb branch moduli and mass parameters, respectively. We just provide the explicit map between Kähler parameters and physical parameters in appendix B. A detailed derivation is given in [29]. We also define $F_{0,1,2}\left(A, M_{i}\right)$ as

$$
\begin{aligned}
F_{0}(\{\boldsymbol{A}\},\{\boldsymbol{M}\}, q)= & \frac{q}{(1-q)^{2}}\left[\sum_{I=1}^{3}\left(-\sum_{i=1}^{10} \frac{A_{I}}{M_{i}}+\sum_{J=1}^{3} \frac{A_{I}}{A_{J}}\right)\right] \\
F_{1}(\{\boldsymbol{A}\},\{\boldsymbol{M}\}, q)= & \frac{q}{(1-q)^{2}}\left[\left(\frac{\tilde{\chi}_{0} A_{1}^{4} \prod_{i=1}^{10}\left(1-A_{1}^{-1} M_{i}\right)}{\left(1-A_{1}^{-1} A_{2}\right)^{2}\left(1-A_{1}^{-1} A_{3}\right)^{2}}+(\text { cyclic })\right)\right. \\
& \left.-\tilde{\chi}_{1}\left(A_{1}+A_{2}+A_{3}\right)^{2}-\tilde{\chi}_{9}\left(A_{1}^{-1}+A_{2}^{-1}+A_{3}^{-1}\right)^{2}+\tilde{\chi}_{2}+\tilde{\chi}_{8}\right] \\
\left.F_{2}(\{\boldsymbol{A}\},\{\boldsymbol{M}\}, q)\right)= & G_{2}(\{\boldsymbol{A}\},\{\boldsymbol{M}\}, q)-\frac{1}{2} F_{1}^{2}(\{\boldsymbol{A}\},\{\boldsymbol{M}\}, q)+\frac{1}{2} F_{1}\left(\left\{\boldsymbol{A}^{2}\right\},\left\{\boldsymbol{M}^{2}\right\}, q^{2}\right),
\end{aligned}
$$

where

$$
\begin{aligned}
G_{2}(\{\boldsymbol{A}\},\{\boldsymbol{M}\}, q)= & \frac{q^{2}}{(1-q)^{4}} \sum_{m=0}^{10} \sum_{n=0}^{10}(-1)^{m+n} \tilde{\chi}_{m} \tilde{\chi}_{n} \\
\times & {\left[\frac{A_{1}{ }^{6-m} A_{2}{ }^{6-n}}{\left(A_{1}-A_{3}\right)^{2}\left(A_{2}-A_{3}\right)^{2}\left(A_{1}-A_{2} q^{-1}\right)^{2}\left(A_{1}-A_{2} q\right)^{2}}\right.} \\
& +\frac{q^{8-n} A_{1}{ }^{12-m-n}}{(1+q)^{2}\left(A_{1}-A_{2}\right)^{2}\left(A_{1}-A_{3}\right)^{2}\left(A_{2}-A_{1} q\right)^{2}\left(A_{3}-A_{1} q\right)^{2}} \\
& \left.+\frac{q^{-6+n} A_{1}{ }^{12-m-n}}{(1+q)^{2}\left(A_{1}-A_{2}\right)^{2}\left(A_{1}-A_{3}\right)^{2}\left(A_{2}-A_{1} q^{-1}\right)^{2}\left(A_{3}-A_{1} q^{-1}\right)^{2}}\right] \\
+ & (\text { cyclic }),
\end{aligned}
$$

and

$$
\tilde{\chi}_{n}=\tilde{\chi}_{0} \sum_{1 \leq i_{1}<i_{2}<\cdots<i_{n} \leq 10} M_{i_{1}} M_{i_{2}} \cdots M_{i_{n}} \quad(n=1,2, \cdots 10), \quad \tilde{\chi}_{0}=\prod_{i=1}^{10} M_{i}^{-\frac{1}{2}} .
$$

A letter "(cyclic)" means two more terms that are obtained by taking a cyclic permutation of $A_{I}(I=1,2,3)$ on the first term. $\left\{\boldsymbol{A}^{2}\right\}$ and $\left\{\boldsymbol{M}^{2}\right\}$ denote the squares of $A_{I}$ and $M_{i}$,

$$
\left\{\boldsymbol{A}^{2}\right\}=\left\{A_{1}^{2}, A_{2}^{2}, A_{3}^{2}\right\}, \quad\left\{\boldsymbol{M}^{2}\right\}=\left\{M_{1}^{2}, M_{2}^{2}, \ldots, M_{10}^{2}\right\} .
$$

\subsection{5-brane web with two O5-planes}

We now compute the partition function based on a 5-brane web with two O5-planes. The corresponding web describes $5 \mathrm{~d} \operatorname{Sp}(2)$ gauge theory with 10 flavors. As it is UV-dual to $6 \mathrm{~d} \operatorname{Sp}(1)$ gauge theory with 10 flavors and a tensor multiplet, a brane configuration for $5 \mathrm{~d}$ $\operatorname{Sp}(2)$ gauge theory with 10 flavors can be obtained as a T-dual version of a Type IIA brane configuration for the 6d theory, which is made out of D6-branes, an O6-plane, and NS5 brane as given in figure 3(a) [30, 31]. The corresponding Type IIB 5-brane configuration for 


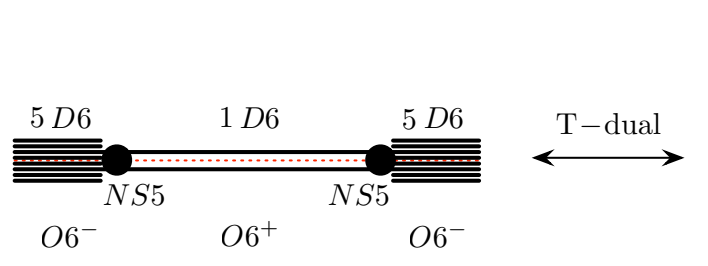

(a)

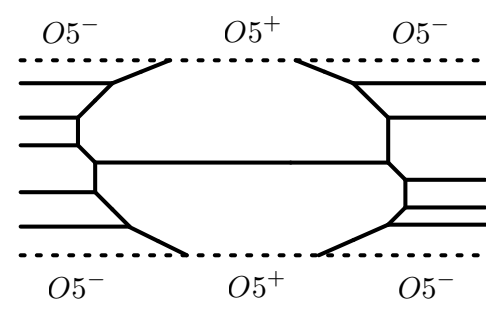

(b)

Figure 3. (a): A Type IIA brane configuration for $6 \mathrm{~d} \mathrm{Sp}(1)$ gauge theory with 10 flavors and a tensor. (b): A Type IIB brane configuration which is T-dual of (a).

(a)

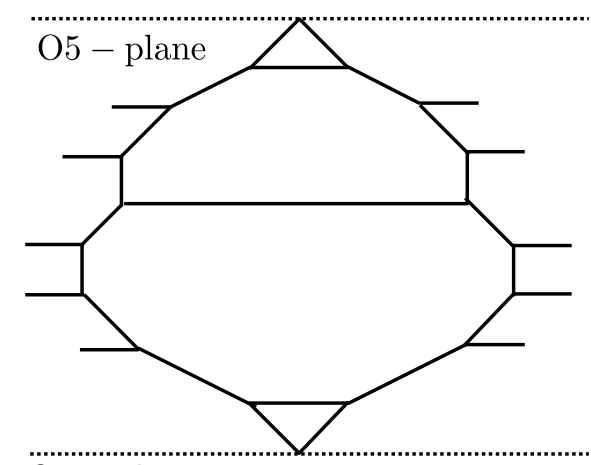

O5 - plane (b)

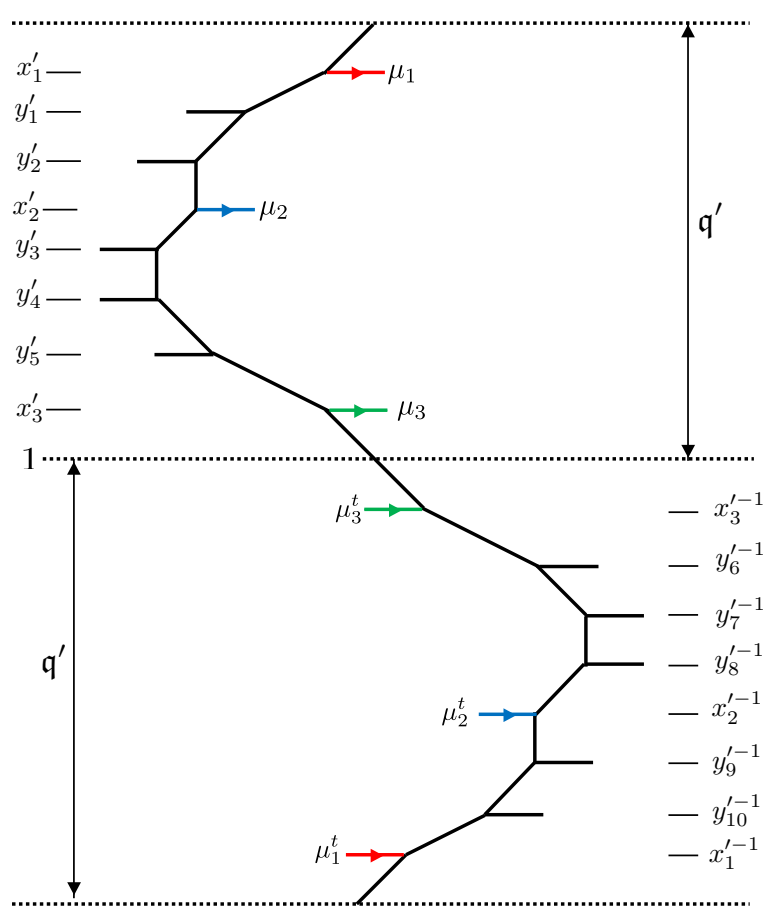

Figure 4. A 5-brane web diagram description of $5 \mathrm{~d} \mathcal{N}=1 \mathrm{Sp}(2)$ gauge theory with 10 flavors. The dashed lines and the solid lines denote the O5-planes and $(p, q) 5$-branes, respectively. The variables $x_{I}^{\prime}(I=1,2,3)$ and $y_{i}^{\prime}(i=1,2, \ldots, 10)$ denote the position of the horizontal legs measured from the middle of the dashed line, and $\mathfrak{q}^{\prime}$ denotes the instanton factor.

$5 \mathrm{~d} \mathrm{Sp}(2)$ gauge theory with 10 flavors is a 5-brane configuration with two O5-planes as given by figure 3(b). As discussed in [34], a 5-brane web diagram in figure 3(b) can be deformed into a 5-brane web diagram given in figure 4(a). Such deformed diagram gives rise to a strip-like diagram when the fundamental configuration is chosen to combine a half from the original diagram and the other from its reflected image due to the O5-plane, as depicted in figure $4(\mathrm{~b})$, where the top of $(1,1)$-brane is gluing to the bottom of $(-1,-1)$-brane by flipping the sign of the charges, i.e. the web diagram is compactified with the period $\mathfrak{q}^{\prime 2}$. This is a crucial 5-configuration which enables one to implement the topological vertex 
method to a 5-brane web with O5-plane(s) [24]. The computation in fact can be done in a straightforward way. With the fundamental configuration as a strip-like diagram given in figure 4(b), one performs the topological vertex computation with the following additional procedures: (i) the framing factor associated with a 5-brane crossing an O5-plane needs to be shifted by 1. (ii) internal edges of the web diagram associated with color D5-branes are glued in the following way: as the configuration on the right hand side of figure 4(b) are reflected, one takes the transpose for the Young diagrams assigned to the internal edges, and then one glues these internal edges together. See appendix C.1 for more details.

Based on the 5-brane configuration in figure 4(b), the partition function for $5 \mathrm{~d} \operatorname{Sp}(2)$ gauge theory with 10 flavors is expressed in terms of a Young diagram sum over $\mu_{1}, \mu_{2}, \mu_{3}$, as

$$
\begin{aligned}
& Z^{\prime \mathrm{Sp}(2)+10 \mathrm{~F}}=\sum_{\mu_{1,2,3}}\left(\frac{\mathfrak{q}^{\prime 2} x_{2}^{\prime} x_{3}^{\prime}}{x^{\prime 4}}\right)^{\left|\mu_{1}\right|}\left(\frac{\prod_{i=1}^{10} y_{i}^{\prime}}{x^{\prime}{ }_{1}^{\prime} x_{2}^{\prime} x_{3}^{\prime}}\right)^{\left|\mu_{2}\right|}\left(\frac{\prod_{i=1}^{10} y_{i}^{\prime}}{x^{\prime}{ }_{1} x_{2}^{\prime} x^{\prime}{ }_{3}^{6}}\right)^{\left|\mu_{3}\right|} f_{\mu_{1}}^{-5} f_{\mu_{2}}^{-5} f_{\mu_{3}}^{-5} \\
& \times \prod_{I=1}^{3}\left(\prod_{i=1}^{10} \frac{\Theta_{\mu_{I} \varnothing}\left(x_{I}^{\prime} y_{i}^{\prime-1}\right)}{\Theta_{\mu_{I} \varnothing}\left(x_{I}^{\prime} y_{i}^{\prime}\right)} \prod_{J=1}^{3} \frac{\Theta_{\mu_{I} \mu_{J}}\left(x_{I}^{\prime} x_{J}^{\prime}\right)}{\Theta_{\mu_{I} \mu_{J}^{t}}\left(x_{I}^{\prime} x_{J}^{\prime-1}\right)}\right),
\end{aligned}
$$

where $f_{\mu}$ is the framing factor defined in (A.5). The positions $x_{I}^{\prime}$ and $y_{i}$ correspond to the fugacities associated with the color D5-branes and those for 10 flavor masses, respectively. For convenience, we have used $\Theta_{\mu \nu}(Q)$ defined as

$$
\Theta_{\mu \nu}(Q)=\prod_{n=0}^{\infty}\left(1-Q \mathfrak{q}^{2 n} q^{i+j-\mu_{i}-\nu_{j}-1}\right)\left(1-Q^{-1} \mathfrak{q}^{2(n+1)} q^{i+j-\mu_{i}^{t}-\nu_{j}^{t}-1}\right)
$$

We note that $\Theta_{\mu \nu}(Q)$ can be expressed as the Jacobi theta functions (A.7) by using the analytic continuation formula [20], and hence it is of periodic structure. Notice that there are three Young diagram sums associated with color D5-branes in (2.12). As discussed earlier, the $5 \mathrm{~d} \mathcal{N}=1 \mathrm{Sp}(N)$ gauge theory with $N_{f}$ flavors is dual to the $5 \mathrm{~d} \mathcal{N}=1 \mathrm{SU}(N+1)_{\kappa}$ gauge theory with $N_{f}$ flavors and the Chern-Simons level $\kappa=N+3-N_{f} / 2$, through some kind of geometric transition $[25,29,35,36]$. This enables us to relate the parameters of the $\operatorname{Sp}(N)$ theory and those of the $\mathrm{SU}(N+1)$ theory. In this case, with a shifting factor $\mathfrak{q}^{1 / 2} \Lambda_{\mathrm{SU}(3)}$ for the Coulomb branch where $\Lambda_{\mathrm{SU}(3)}=\prod_{i=1}^{10} M_{i}^{-1 / 4}$, the parameter map between the $\mathrm{Sp}(2)$ gauge theory with 10 flavors $\left(x_{I}^{\prime}, y_{i}^{\prime}\right)$ and the $\mathrm{SU}(3)_{0}$ gauge theory with 10 flavors $\left(A_{I}, M_{i}\right)$ is given by $[29]$

$$
\begin{aligned}
& x_{I}^{\prime}=\mathfrak{q}^{1 / 2} A_{I} \Lambda_{\mathrm{SU}(3)} \quad(I=1,2,3), \\
& y_{i}^{\prime}=\mathfrak{q}^{1 / 2} M_{i} \Lambda_{\mathrm{SU}(3)} \quad(i=1, \cdots, 10) \text {, }
\end{aligned}
$$

where the $\mathrm{SU}(3)$ Coulomb branch moduli $A_{I}$ satisfy $A_{1} A_{2} A_{3}=1$. We note that the instanton factors for these two dual theories are same $\mathfrak{q}^{\prime}=\mathfrak{q}$, as they are associated with the compactification radius. 
With the $\mathrm{SU}(3)$ gauge theory parametrization, we find the partition function of $\mathrm{SU}(3)_{0}$ gauge theory with 10 flavors in a symmetric form,

$$
\begin{aligned}
Z^{\prime \mathrm{Sp}(2)+10 \mathrm{~F}}= & Z^{\text {extra }} \sum_{\mu_{1,2,3}}\left(\mathfrak{q} \Lambda_{\mathrm{SU}(3)}^{-2} A_{1}^{-5}\right)^{\left|\mu_{1}\right|}\left(\mathfrak{q} \Lambda_{\mathrm{SU}(3)}^{-2} A_{2}^{-5}\right)^{\left|\mu_{2}\right|}\left(\mathfrak{q} \Lambda_{\mathrm{SU}(3)}^{-2} A_{3}^{-5}\right)^{\left|\mu_{3}\right|} f_{\mu_{1}}^{-5} f_{\mu_{2}}^{-5} f_{\mu_{3}}^{-5} \\
& \times \prod_{I=1}^{3}\left(\prod_{i=1}^{10} \frac{\Theta_{\mu_{I} \varnothing}\left(A_{I} M_{i}^{-1}\right)}{\Theta_{\mu_{I} \varnothing}\left(\mathfrak{q} A_{I} M_{i} \Lambda_{\mathrm{SU}(3)}^{2}\right)} \prod_{J=1}^{3} \frac{\Theta_{\mu_{I} \mu_{J}}\left(\mathfrak{q} A_{I} A_{J} \Lambda_{\mathrm{SU}(3)}^{2}\right)}{\Theta_{\mu_{I} \mu_{J}^{t}}\left(A_{I} A_{J}^{-1}\right)}\right)
\end{aligned}
$$

where $Z^{\text {extra }}$ is an Coulomb branch moduli independent part which is given by

$$
Z^{\text {extra }}=\prod_{i, j=1}^{\infty}\left[\frac{1}{\left(q^{i+j-1} \mathfrak{q} ; \mathfrak{q}\right)_{\infty}^{10}} \times \frac{\prod_{1 \leq i, j \leq 5} \Theta_{\phi \varnothing}\left(\mathfrak{q} M_{i} M_{j+5} \Lambda_{\mathrm{SU}(3)}^{2}\right)}{\prod_{1 \leq i<j \leq 5} \Theta_{\varnothing \varnothing}\left(M_{i} M_{j}^{-1}\right) \Theta_{\varnothing \varnothing}\left(M_{i+5} M_{j+5}^{-1}\right)}\right]
$$

where $(a ; \mathfrak{q})_{\infty}=\prod_{k=0}^{\infty}\left(1-a \mathfrak{q}^{k}\right)$ is so-called Pochhammer symbol. There are in fact more Coulomb branch independent part in (2.15), as a whole we call them the extra factor. When obtaining the partition function, we mod out such an extra factor from the topological string partition function. From here on, we neglect the extra factor.

By expressing the partition function (2.15) as an expansion of the instanton factor $\mathfrak{q}$, we can write the partition function for $\mathrm{SU}(3)_{0}$ gauge theory with 10 flavors as the Plethystic exponential, it takes the following form

$$
Z^{\prime \mathrm{Sp}(2)+10 \mathrm{~F}}=\mathrm{PE}\left[F_{0}(\{\boldsymbol{A}\},\{\boldsymbol{M}\})+F_{1}(\{\boldsymbol{A}\},\{\boldsymbol{M}\}) \mathfrak{q}+F_{2}(\{\boldsymbol{A}\},\{\boldsymbol{M}\}) \mathfrak{q}^{2}+\mathcal{O}\left(\mathfrak{q}^{3}\right)\right]
$$

where $F_{n}(\{\boldsymbol{A}\},\{\boldsymbol{M}\})$ are exactly the same as those obtained from Tao diagram (2.8a), (2.8b), and (2.8c) since they are the dual to each other, although the partition functions $Z^{\mathrm{SU}(3)+10 \mathrm{~F}}$ and $Z^{\mathrm{Sp}(2)+10 \mathrm{~F}}$ are derived from completely different diagrams. ${ }^{3}$ Due to computational complication, we only presented terms of quadratic order in $\mathfrak{q}$, but can be checked the equivalence to higher orders.

\section{Defect partition function of E-strings}

In this section, we consider defect insertions to the E-string theory, from the 5-brane perspective. Through the geometric transitions, we can introduce a codimension 2 defect which is a topological brane wrapping on the Lagrange submanifold in the topological string theory [15]. We consider the partition function of the topological string in the presence of the topological brane, which we call, for short, the defect partition function.

To obtain the defect partition function ${ }^{4}$ for the E-string theory, we utilize geometric transition [17]. The procedure is to set the Kähler parameters associated with Higgsing $Q$ to the $M$-th power of the exponential of the string coupling constant, $Q=q^{M}$, where $M$ is the number of the topological branes. In the context of the gauge theory, when $M=0$, it becomes the usual Higgsing, as $Q=1$ reduces the number of Coulomb branch moduli.

\footnotetext{
${ }^{3}$ As we have already mentioned, these partition functions are the same up to extra factor.

${ }^{4}$ We note that one can also introduce the holonomy matrix for the 5-brane in the topological vertex formalism as has been done e.g., in [23].
} 


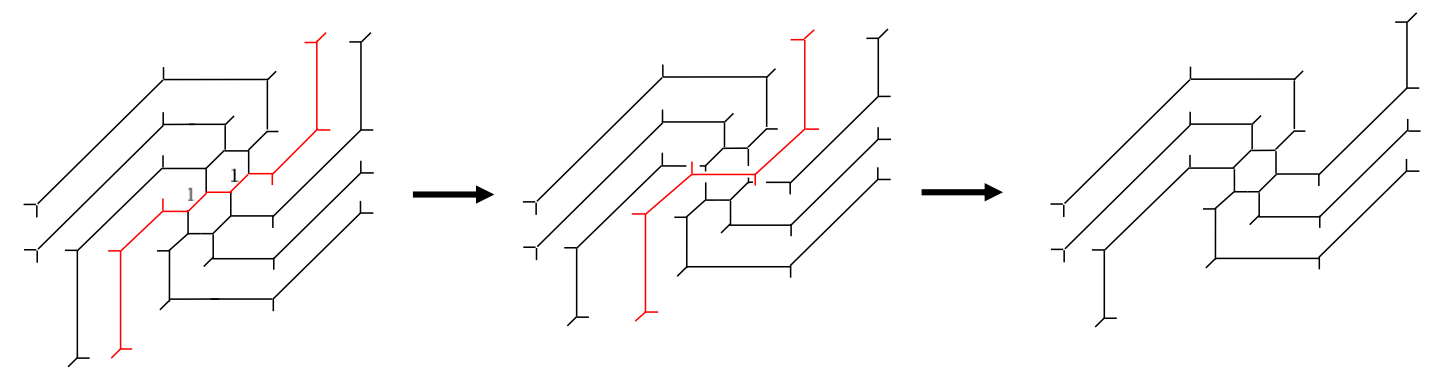

Figure 5. Higgsing in a Tao diagram for SU(3) theory with 10 flavors. Since the framing denoted by the red lines decouples from the remaining web diagram, the part of the summation of the Young diagram is decoupled from other summations.

When $M>0$, it corresponds to the defects in the E-strings. We call such a procedure for introducing the defects a defect Higgsing. More concretely, when we consider the $5 \mathrm{~d}$ $\mathcal{N}=1 \operatorname{Sp}(N)$ gauge theory with $2 N+6$ flavors for $N \geq 2$, the defect Higgsing means

$$
\operatorname{Sp}(N)+(2 N+6) \text { flavors } \rightarrow \operatorname{Sp}(N-1)+(2 N+4) \text { flavors }+ \text { defects. }
$$

Or, from the dual theory point of view, $\mathrm{SU}(N+1)$ gauge theory with the same number of the flavors, it is

$$
\mathrm{SU}(N+1)+(2 N+6) \text { flavors } \rightarrow \mathrm{SU}(N)+(2 N+4) \text { flavors }+ \text { defects. }
$$

In the following, we choose $N=2$ but $M>0$ arbitrary, which is relevant for $M$ defects in the E-strings. We explicitly compute the defect partition function for the E-string based on 5-brane webs by implementing the defect Higgsings.

\subsection{Usual Higgsing on 5-brane webs}

Before we consider the defect Higgsing, we first review the usual Higgsing procedure in 5 -brane configurations with or without O5-planes. As done in the previous section, we consider $5 \mathrm{~d} \mathcal{N}=1 \mathrm{SU}(3)$ gauge theory with 10 flavors and then consider $\mathcal{N}=1 \mathrm{Sp}(2)$ gauge theory with 10 flavors. We will show that one obtains $\mathrm{SU}(2)$ gauge theory with 8 flavors, as a result of the Higgsing on both theories. As discussed earlier, the 5-brane web for SU(3) gauge theory with 10 flavors is a Tao diagram given in figure 1(a). To apply a Higgsing to the SU(2) theory with 8 flavors, we consider a strip consisting of a color D5-brane and two flavor branes that are connected. Unlike the typical 5-brane web, for a Tao diagram, such a strip is a spiral strip as painted in red in figure 5(a). A usual Higgsing is then realized by assigning the relevant Kähler parameters to 1, so that such a spiral string can be Higgsed away, reducing the dimension of the Coulomb branch by one and the number of flavors by two. A diagrammatic procedure is depicted in figure 5 .

For implementing the Higgsing in the topological vertex calculation, as before, we can consider half of the diagram in figure 1(b). More specifically, in figure $2(\mathrm{a})$, say $\tilde{Q}_{2}$ is the Kähler parameter associated with the Higgsing, then we set $\tilde{Q}_{2}=1$. We expect that the 
part involving the summation over the Young diagram $\mu_{2}$ associated with the edges that are Higgsed is factorized in the partition function. In particular, the contribution involving the Young diagram $\mu_{2}$ in the building block $Z_{\text {middle }}$ in $(2.5 \mathrm{a})$ becomes

$$
\frac{R_{\nu_{1} \mu_{2}}\left(Q_{1} \tilde{Q}_{1} Q_{2}\right) R_{\nu_{2} \mu_{2}}\left(Q_{2}\right) R_{\nu_{3} \mu_{3}}\left(Q_{3}\right) R_{\nu_{3} \nu_{4}}\left(Q_{3} \tilde{Q}_{3} Q_{4}\right) R_{\mu_{2} \nu_{3}}(1) R_{\mu_{2} \phi}\left(Q_{3} \tilde{Q}_{3}\right)}{R_{\nu_{1} \nu_{3}}\left(Q_{1} \tilde{Q}_{1} Q_{2}\right) R_{\nu_{2} \nu_{3}}\left(Q_{2}\right) R_{\nu_{3} \nu_{3}}(1) R_{\nu_{3} \phi}\left(Q_{3} \tilde{Q}_{3}\right) R_{\mu_{2} \mu_{3}}\left(Q_{3}\right) R_{\mu_{2} \nu_{4}}\left(Q_{3} \tilde{Q}_{3} Q_{4}\right)}
$$

which vanishes unless $\mu_{2}=\nu_{3}$ due to the factor $R_{\mu_{2} \nu_{3}}(1),{ }^{5}$

$$
R_{\mu_{2} \nu_{3}}(1)=\delta_{\mu_{2}, \nu_{3}} R_{\mu_{2} \mu_{2}}(1)
$$

so that (3.3) becomes 1 under the Higgsing. By combining another half building blocks, the summation of $\mu_{2}$ is decoupled from other summations, as we expected,

$$
Z^{\mathrm{SU}(3)+10 \mathrm{~F}} \rightarrow Z_{\text {Framing }}^{\text {Tao }} Z^{\mathrm{SU}(2)+8 \mathrm{~F}}
$$

where $Z^{\mathrm{SU}(2)+8 \mathrm{~F}}$ is the partition function of $\mathrm{SU}(2)$ gauge theory with 8 flavors [22],

$$
Z^{\mathrm{SU}(2)+8 \mathrm{~F}}=\sum_{\mu_{1,3},\left\{\nu_{i}\right\}} Z_{\text {glue }}\left(Q_{b_{1}}, \mu_{1}\right) Z_{\text {glue }}\left(Q_{b_{3}}, \mu_{3}\right) Z_{\text {half } 1}^{\mathrm{SU}(2)+8 \mathrm{~F}} Z_{\text {half } 2}^{\mathrm{SU}(2)+8 \mathrm{~F}},
$$

with

$$
\begin{aligned}
& Z_{\text {half } 1}^{\mathrm{SU}(2)+8 \mathrm{~F}}=\sum_{\nu_{1,2,4}} Z_{\text {middle }}^{\mathrm{SU}(2)}\left(\nu_{1}, \nu_{2}, \varnothing, \mu_{1}, \mu_{3}, \nu_{4},\{\boldsymbol{Q}\},\{\tilde{\boldsymbol{Q}}\}\right) \\
& \times \prod_{l=1,2} Z_{\text {spiral } 1}\left(\left\{\boldsymbol{Q}^{(l)}\right\}, \nu_{l}\right) \times Z_{\text {spiral } 2}\left(\left\{\boldsymbol{Q}^{(4)}\right\}, \nu_{4}\right), \\
& \left.Z_{\text {middle }}^{\mathrm{SU}(2)+8 \mathrm{~F}}\left(\mu_{1}, \mu_{3}, \mu_{4}, \nu_{1}, \nu_{2}, \nu_{4},\{\boldsymbol{Q}\},\{\tilde{\boldsymbol{Q}}\}\right\}\right) \\
& =\frac{\prod_{\substack{1 \leq i \leq j \leq 4 \\
(i \neq 2, j \neq 3)}} R_{\mu_{i} \nu_{j}}\left(Q_{j} \prod_{k=1}^{j-1} Q_{k} \tilde{Q}_{k}\right) \prod_{\substack{1 \leq i<j \leq 4 \\
i \neq 3, j \neq 2)}} R_{\nu_{i} \mu_{j}}\left(\tilde{Q}_{i} \prod_{k=i+1}^{j-1} Q_{k} \tilde{Q}_{k}\right)}{\prod_{\substack{1 \leq i \leq j \leq 4 \\
(i, j \neq 2)}} R_{\mu_{i} \mu_{j}}\left(\prod_{k=1}^{j-1} Q_{k} \tilde{Q}_{k}\right) \prod_{\substack{1 \leq i<j \leq 4 \\
(i, j \neq 3)}} R_{\nu_{i} \nu_{j}}\left(\prod_{k=i}^{j-1} Q_{k+1} \tilde{Q}_{k}\right)}, \\
& Z_{\text {half } 2}^{\mathrm{SU}(2)+8 \mathrm{~F}}=Z_{\text {half } 1}^{\mathrm{SU}(2)+8 \mathrm{~F}}\left(M_{i} \leftrightarrow M_{i+5}^{-1} ; A_{1} \leftrightarrow A_{3}^{-1}\right),
\end{aligned}
$$

and $Z_{\text {Framing }}^{\text {Tao }}$ can be interpreted as the contribution coming from the framing denoted by the red-colored strips in figure 5 , which is given by

$$
Z_{\text {Framing }}^{\text {Tao }}=\sum_{\mu_{2}} Z_{\text {glue }}\left(Q_{b_{2}}, \mu_{2}\right) Z_{\text {spiral } 1}\left(\left\{\boldsymbol{Q}^{(3)}\right\}, \mu_{2}\right) R_{\mu_{2} \mu_{2}}(1) .
$$

Now we consider the Higgsing from the 5 -brane web in figure 6 for the $5 \mathrm{~d} \mathcal{N}=1 \mathrm{Sp}(2)$ gauge theory with 10 flavors. ${ }^{6}$ The Higgsing procedure is almost the same as that on the Tao diagram. We align a color brane and two flavor branes, for instance,

$$
A_{2}=M_{3}=M_{8} \text {. }
$$

\footnotetext{
${ }^{5}$ See also [37] for refined case.

${ }^{6}$ This Higgsing is also considered in [38] in the context of the elliptic genus.
} 

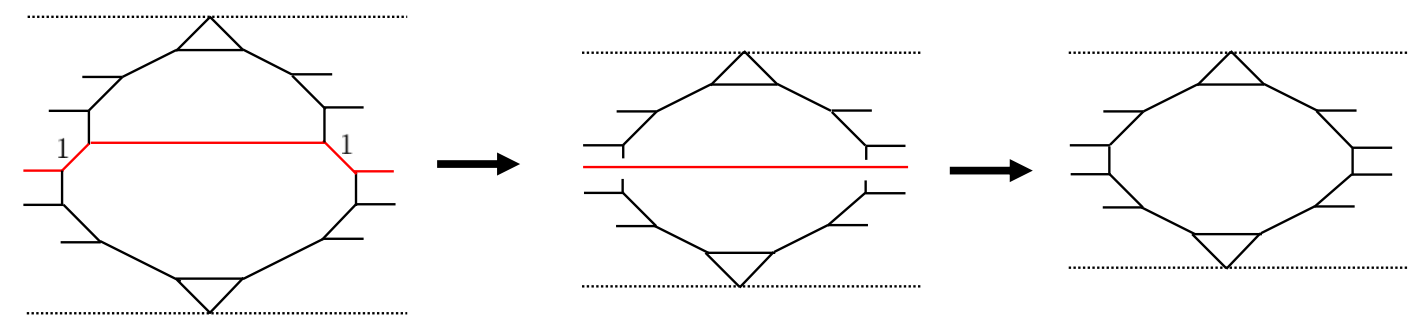

Figure 6. A web diagram description of a usual Higgsing. By setting the Kähler parameters to 1 , one can equate the Coulomb branch parameter and the fundamental masses. The D5-brane in red then can be taken to the Higgs branch directions. The resulting web diagram gives $5 \mathrm{~d} \mathcal{N}=1$ $\mathrm{Sp}(1)$ gauge theory with 8 flavors.

It is also straightforward to see that the partition function (2.12) vanishes unless the associated Young diagram is $\mu_{2}=\varnothing$, and hence it yields that the partition function (2.12) reduces to

$$
Z^{\prime \mathrm{Sp}(2)+10 \mathrm{~F}} \rightarrow Z_{\mathrm{Framing}}^{\mathrm{O} 5} Z^{\prime \mathrm{Sp}(1)+8 \mathrm{~F}}
$$

Here $Z^{\prime S p(1)+8 F}$ is the partition function for the $5 \mathrm{~d} \mathcal{N}=1 \mathrm{Sp}(1)$ gauge theory with 8 flavors, given by

$$
Z^{\prime \mathrm{Sp}(1)+8 \mathrm{~F}}=\sum_{\mu_{1,3}}\left(\mathfrak{q} \Lambda_{\mathrm{SU}(2)}^{-2} A_{1}^{-4}\right)^{\left|\mu_{1}\right|}\left(\mathfrak{q} \Lambda_{\mathrm{SU}(2)}^{-2} A_{3}^{-4}\right)^{\left|\mu_{3}\right|} f_{\mu_{1}}^{-4} f_{\mu_{3}}^{-4} Z_{\mathrm{build}}^{\mathrm{Sp}(1)+8 \mathrm{~F}}
$$

where

$$
Z_{\text {build }}^{\mathrm{Sp}(1)+8 \mathrm{~F}}:=\prod_{I=1,3}\left(\prod_{i \in \mathcal{I}} \frac{\Theta_{\mu_{I} \varnothing}\left(A_{I} M_{i}^{-1}\right)}{\Theta_{\mu_{I} \varnothing}\left(\mathfrak{q} A_{I} M_{i} \Lambda_{\mathrm{SU}(2)}^{2}\right)} \prod_{J=1,3} \frac{\Theta_{\mu_{I} \mu_{J}}\left(\mathfrak{q} A_{I} A_{J} \Lambda_{\mathrm{SU}(2)}^{2}\right)}{\Theta_{\mu_{I} \mu_{J}^{t}}\left(A_{I} A_{J}^{-1}\right)}\right)
$$

with $\Lambda_{\mathrm{SU}(2)}=\prod_{i \in \mathcal{I}} M_{i}^{-1 / 4}$ and $\mathcal{I}=\{1,2,4,5,6,7,9,10\}$. The factor $Z_{\text {Framing }}^{\mathrm{O} 5}$ is an extra factor which comes from the contribution of the framing denoted by the red line in figure 6 ,

$$
Z_{\mathrm{Framing}}^{\mathrm{O} 5}=\prod_{i, j=1}^{\infty}\left(1-q^{i+j-1}\right)
$$

By rescaling the Coulomb moduli parameters and the mass parameters as,

$$
A_{1} \rightarrow M_{3}^{-1 / 2} A_{1}, \quad A_{3} \rightarrow M_{3}^{-1 / 2} A_{3}, \quad M_{i} \rightarrow M_{3}^{-1 / 2} M_{i}(i \neq 3,8),
$$

we see that (3.11) agrees with the partition function directly calculated from the web diagram of $5 \mathrm{~d} \mathcal{N}=1 \mathrm{SU}(2)$ with 8 flavors discussed in [24].

\subsection{Defect Higgsing on 5-brane webs}

From here on, we consider a 5-brane system with defect D3-branes, which can be introduced in a similar way as for usual Higgsings. As discussed, the defect Higgsing is the Higgsing by 
(a)

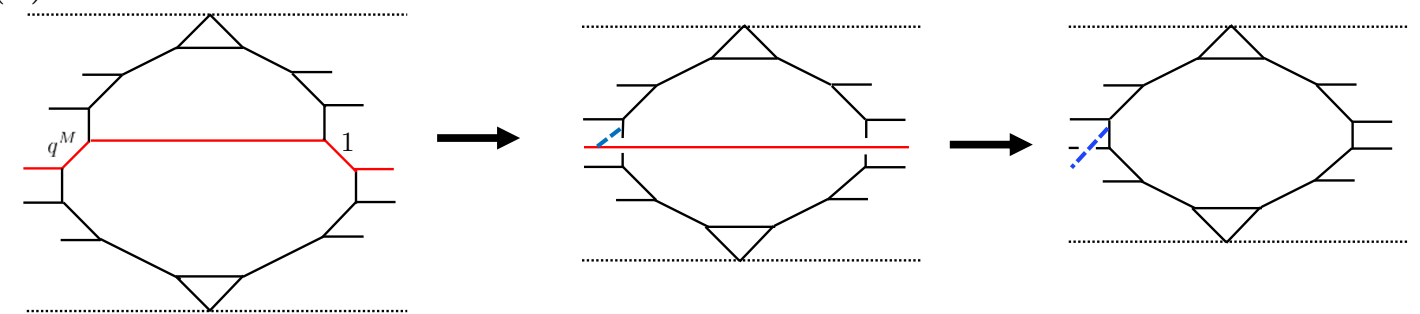

(b)

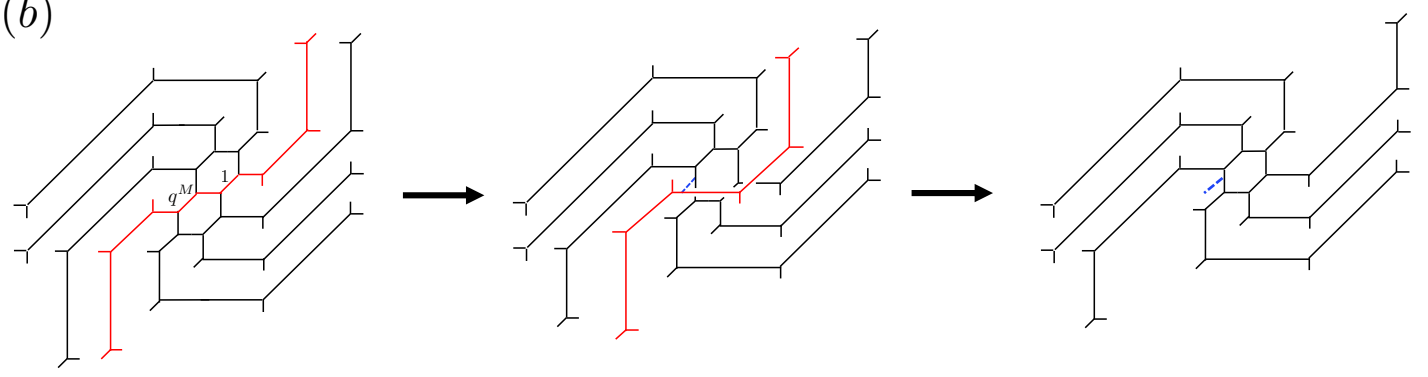

Figure 7. The 5-branes with the defect introduced by defect Higgsing. The blue dashed lines denote the defects.

setting the associated Kähler parameter not 1 but $q^{M}[4,15,39]$. Here, $M$ is the number of defect D3-branes, and hence the defect Higgsing becomes a usual Higgsing when $M=0$. For instance, in figure 7(a), 5-brane web for the $5 \mathrm{~d} \operatorname{Sp}(2)$ theory with 10 flavors can be used to describe the $5 \mathrm{~d} \operatorname{Sp}(1)$ theory with 8 flavors and defects, where the 5-branes painted in red are defect Higgsed. The associated Kähler parameter is set to be $q^{M}$, while the other Kähler parameter is just treated as a usual Higgsing. After having defect Higgsed, we denote the defect Higgsed part as a (blue) dotted line, representing $M$ D3-branes. Likewise, such defect Higgsing can be performed in a Tao diagram in the same way, as depicted in figure 7(b).

Implementing the defect Higgsings to the partition function is straightforward. For the partition functions that we obtained from topological vertex based on the web diagrams either with O5-planes or with the Tao diagram, in the previous section, we first set one of the Coulomb branch moduli $A_{2}$ to

$$
A_{2}=q^{M} z=M_{8}
$$

where we defined $z=M_{3}$ as a defect moduli. When $M=0$, it reduces to (3.9), as expected. As the computation is similar to the usual Higgsing, we summarize the results for both cases.

Defect Higgsing on SU(3) 5-brane web. Let us first consider the defect Higgsing on 5-brane web for $\mathcal{N}=1 \mathrm{SU}(3)$ gauge theory with 10 flavors, given in figure 7(b). For the partition function (3.5) obtained from the Tao web diagram, the defect Higgsing on SU(3) 
gauge theory with 10 flavors yields

$$
\begin{aligned}
Z^{\mathrm{SU}(3)+10 \mathrm{~F}} \rightarrow & \sum_{\mu_{1,3},\left\{\nu_{i}\right\}} Z_{\text {glue }}\left(Q_{b_{1}}, \mu_{1}\right) Z_{\text {glue }}\left(Q_{b_{3}}, \mu_{3}\right) Z_{\text {half } 1}^{\mathrm{SU}(2)+8 \mathrm{~F}} Z_{\text {half } 2}^{\mathrm{SU}(2)+8 \mathrm{~F}} \\
& \times Z_{\text {glue }}\left(Q_{b_{2}}, \nu_{3}\right) Z_{\text {spiral } 1}\left(\left\{\boldsymbol{Q}^{(3)}\right\}, \nu_{3}\right) Z_{\text {defect Tao }} \\
= & : Z^{\mathrm{SU}(2)+8 \mathrm{~F}, \text { defect }}
\end{aligned}
$$

where

$$
\begin{aligned}
Z_{\text {defect Tao }}= & \frac{R_{\nu_{1} \nu_{3}}\left(Q_{1} \tilde{Q}_{1} Q_{2}\right) R_{\nu_{2} \nu_{3}}\left(Q_{2}\right) R_{\nu_{3} \nu_{3}}\left(q^{M}\right) R_{\nu_{3} \mu_{3}}\left(Q_{3}\right)}{R_{\nu_{1} \nu_{3}}\left(q^{M} Q_{1} \tilde{Q}_{1} Q_{2}\right) R_{\nu_{2} \nu_{3}}\left(q^{M} Q_{2}\right) R_{\nu_{3} \nu_{3}}(1)} \\
& \times \frac{R_{\nu_{3} \nu_{4}}\left(Q_{3} \tilde{Q}_{3} Q_{4}\right) R_{\nu_{3} \varnothing}\left(q^{M} Q_{3} \tilde{Q}_{3}\right) R_{\mu_{1} \nu_{3}}\left(q^{M} \tilde{Q}_{1} Q_{2}\right)}{R_{\nu_{3} \mu_{3}}\left(q^{M} Q_{3}\right) R_{\nu_{3} \nu_{4}}\left(q^{M} Q_{3} \tilde{Q}_{3} Q_{4}\right) R_{\nu_{3} \varnothing}\left(Q_{3} \tilde{Q}_{3}\right) R_{\mu_{1} \nu_{3}}\left(\tilde{Q}_{1} Q_{2}\right)},
\end{aligned}
$$

and we have rescaled some variables as follows,

$$
A_{I} \rightarrow q^{-M / 2} z^{-1 / 2} A_{I},(I=1,3),
$$

to obtain the traceless condition of $\mathrm{SU}(2), A_{1} A_{3}=1$. This then naturally shifts other mass parameters

$$
M_{i} \rightarrow q^{-M / 2} z^{-1 / 2} M_{i},(i \neq 3,8) .
$$

Defect Higgsing on $\mathrm{Sp}(2)$ 5-brane web. Next, we consider the defect Higgsing on 5-brane web for $\mathcal{N}=1 \mathrm{Sp}(2)$ gauge theory with 10 flavors, given in figure 7(a). For the partition function (2.15) obtained from the 5-brane web with O5-planes, the defect Higgsing on the $\operatorname{Sp}(2)$ gauge theory with 10 flavors yields

$$
\begin{aligned}
Z^{\prime \mathrm{Sp}(2)+10 \mathrm{~F}} \rightarrow & \prod_{i=1}^{4} \frac{\Theta_{\varnothing \varnothing}\left(q^{M} z M_{i}^{-1}\right) \Theta_{\varnothing \varnothing}\left(\mathfrak{q} z M_{i+4} \Lambda_{\mathrm{SU}(2)}^{2}\right)}{\Theta_{\varnothing \varnothing}\left(z M_{i}^{-1}\right) \Theta_{\varnothing \varnothing}\left(\mathfrak{q} z q^{M} M_{i+4} \Lambda_{\mathrm{SU}(2)}^{2}\right)} \\
& \times \sum_{\mu_{1,3}}\left(\mathfrak{q} q^{M / 2} z \Lambda_{\mathrm{SU}(2)}^{-2} A_{1}^{-4}\right)^{\left|\mu_{1}\right|}\left(\mathfrak{q} q^{M / 2} z \Lambda_{\mathrm{SU}(2)}^{-2} A_{3}^{-4}\right)^{\left|\mu_{3}\right|} f_{\mu_{1}}^{-4} f_{\mu_{3}}^{-4} \\
& \times Z_{\text {build }}^{\text {Sp }(1)+8 \mathrm{~F}}\left(\Lambda_{\mathrm{SU}(2)} \rightarrow q^{M / 4} \Lambda_{\mathrm{SU}(2)}\right) Z^{\text {defect O5 }} \\
= & : Z^{\text {Sp }(1)+8 \mathrm{~F}, \text { defect }}
\end{aligned}
$$

where $Z_{\text {build }}^{\prime \mathrm{Sp}(1)+8 \mathrm{~F}}$ is defined in (3.12) with the replacement $\Lambda_{\mathrm{SU}(2)} \rightarrow q^{M / 4} \Lambda_{\mathrm{SU}(2)}$, and

$$
\begin{aligned}
& Z^{\text {defect O5 }} \\
& =\frac{\Theta_{\mu_{1} \varnothing}\left(q^{-M / 2} z^{-3 / 2} A_{1}\right) \Theta_{\mu_{3} \varnothing}\left(q^{-M / 2} z^{-3 / 2} A_{3}\right) \Theta_{\mu_{1} \varnothing}\left(\mathfrak{q} q^{M} z^{3 / 2} A_{1} \Lambda_{\mathrm{SU}(2)}^{2}\right) \Theta_{\mu_{3} \varnothing}\left(\mathfrak{q} q^{M} z^{3 / 2} A_{3} \Lambda_{\mathrm{SU}(2)}^{2}\right)}{\Theta_{\mu_{1} \varnothing}\left(q^{-3 M / 2} z^{-3 / 2} A_{1}\right) \Theta_{\mu_{3} \varnothing}\left(q^{-3 M / 2} z^{-3 / 2} A_{3}\right) \Theta_{\mu_{1} \varnothing}\left(\mathfrak{q} z^{3 / 2} A_{1} \Lambda_{\mathrm{SU}(2)}^{2}\right) \Theta_{\mu_{3} \varnothing}\left(\mathfrak{q} z^{3 / 2} A_{3} \Lambda_{\mathrm{SU}(2)}^{2}\right)} .
\end{aligned}
$$

We note that though two defect partition functions (3.16) and (3.20) look different, it is straightforward to see that they are equivalent when we expand as $\mathfrak{q}$ and $A_{1}$.

When expanding the partition function as a series of the instanton factor and the defect moduli, the partition function is decomposed into two parts: one is the defect moduli independent part which hence depends only on the Coulomb moduli and mass parameters. 
We denote it by $F_{n}^{\text {defect }}(A)$. The other is the part that explicitly depends on the defect moduli, which we denote by $D_{n}^{\text {defect }}(z, A)$, up to an extra factor,

$$
Z^{\prime \mathrm{Sp}(1)+8 \mathrm{~F}, \text { defect }}=\mathrm{PE}\left[\sum_{n=0}^{\infty} \mathfrak{q}^{n}\left(F_{n}^{\text {defect }}(A)+D_{n}^{\text {defect }}(z, A)\right)\right],
$$

where $A=A_{1}$. Up to 2-instanton orders, $F_{n}^{\text {defect }}(A)$ and $D_{n}^{\text {defect }}(x, A)$ take the following forms. In terms of the $\mathrm{U}(8)$ characters,

$$
\begin{aligned}
\chi_{n} & =\chi_{0} \sum_{\substack{1 \leq i_{1}<i_{2}<\ldots<i_{n} \leq 10 \\
i_{m} \in \mathcal{I}}} M_{i_{1}} M_{i_{2}} \ldots M_{i_{n}}, \quad n=1,2, \ldots, 8, \\
\chi_{0} & =\prod_{i \in \mathcal{I}} M_{i}^{-1 / 2},
\end{aligned}
$$

they are given by, at $\mathfrak{q}^{0}$ order,

$$
\begin{aligned}
F_{0}^{\text {defect }}(A) & =\frac{q}{(1-q)^{2}}\left(-A\left(\chi_{1} \chi_{8}+\chi_{0} \chi_{7}\right)+2 A^{2}\right), \\
D_{0}^{\text {defect }}(z, A) & =\frac{q\left(q^{M / 2}-q^{-M / 2}\right)}{(1-q)^{2}} A\left(q^{M} z^{3 / 2}-q^{-M} z^{-3 / 2}\right),
\end{aligned}
$$

and, at $\mathfrak{q}^{1}$ order,

$$
\begin{gathered}
F_{1}^{\text {defect }}(A)=-\frac{q}{(1-q)^{2}}\left(q^{M / 2}\left(\chi_{1}+\chi_{3}\right)+q^{-M / 2}\left(\chi_{5}+\chi_{7}\right)\right) A+\mathcal{O}\left(A^{2}\right), \\
D_{1}^{\text {defect }}(z, A)=\frac{q\left(q^{M / 2}-q^{-M / 2}\right)}{(1-q)^{2}}\left[\left(q^{9 M / 2} z^{9 / 2} \chi_{0}-q^{-9 M / 2} z^{-9 / 2} \chi_{8}\right)-\left(q^{3 M} z^{3} \chi_{1}-q^{-3 M} z^{-3} \chi_{7}\right)\right. \\
\left.\quad+\left(q^{3 M / 2} z^{3 / 2} \chi_{2}-q^{-3 M / 2} z^{-3 / 2} \chi_{6}\right)+\mathcal{O}\left(z^{15 / 2}\right)\right] A+\mathcal{O}\left(A^{2}\right),
\end{gathered}
$$

and at $\mathfrak{q}^{2}$ order,

$$
\begin{aligned}
F_{2}^{\text {defect }}(A)= & -\frac{q}{(1-q)^{2}}\left[q^{M}\left(\chi_{0} \chi_{5}+\chi_{0} \chi_{7}+\chi_{1} \chi_{8}\right)+q^{-M}\left(\chi_{3} \chi_{8}+\chi_{0} \chi_{5}+\chi_{1} \chi_{8}\right)\right. \\
& \left.-2\left(q+q^{-1}+5\right)\left(\chi_{0} \chi_{7}+\chi_{1} \chi_{8}\right)-\left(\chi_{1} \chi_{6}+\chi_{2} \chi_{7}-7\left(\chi_{0} \chi_{7}+\chi_{1} \chi_{8}\right)\right)\right] A \\
& +\mathcal{O}\left(A^{2}\right), \\
D_{2}^{\text {defect }}(z, A)= & \frac{q\left(q^{M / 2}-q^{-M / 2}\right)}{(1-q)^{2}}\left[q^{8 M} \chi_{0}^{2} z^{15 / 2}-q^{-8 M} \chi_{0}^{2} z^{-15 / 2}\right. \\
& -q^{13 M / 2} \chi_{0} \chi_{1} z^{6}+q^{-13 M / 2} \chi_{7} \chi_{8} z^{-6}+q^{5 M} \chi_{0} \chi_{2} z^{9 / 2}-q^{-5 M} \chi_{6} \chi_{8} z^{-9 / 2} \\
& -\left(q^{7 M / 2} \chi_{0} \chi_{3}+q^{5 M / 2} \chi_{0} \chi_{7}\right) z^{3}+\left(q^{-7 M / 2} \chi_{5} \chi_{8}+q^{-5 M / 2} \chi_{1} \chi_{8}\right) z^{-3} \\
& +\left(q^{2 M}\left(-1+\chi_{0} \chi_{4}\right)+q^{M}\left(2 q+2 q^{-1}+2+\chi_{0} \chi_{6}+\chi_{1} \chi_{7}\right)\right) z^{3 / 2} \\
& -\left(q^{2 M}\left(-1+\chi_{8} \chi_{4}\right)+q^{M}\left(2 q+2 q^{-1}+2+\chi_{2} \chi_{8}+\chi_{1} \chi_{7}\right)\right) z^{-3 / 2} \\
& \left.+\mathcal{O}\left(z^{21 / 2}\right)\right] A+\mathcal{O}\left(A^{2}\right) .
\end{aligned}
$$


Here we note that the global symmetry is seemingly broken through the defect Higgsing, as the defect partition function is expressed in terms of the $\mathrm{U}(8)$ characters. However, recall that the usual Higgsing from the $\mathrm{SU}(3)$ gauge theory with 10 flavors to the $\mathrm{SU}(2)$ gauge theory with 8 flavors preserves an $E_{8}$ symmetry, and such enhanced global symmetry can be seen suitably combining flavors masses and instanton fugacity such that they form the characters of the enhanced global symmetry. To make the global symmetry of the theory in the presence of the defects manifest, we consider the elliptic genus.

\subsection{Defect Higgsing in elliptic genus and global symmetry}

We compute the elliptic genus of E-strings with defect by utilizing the defect Higgsing. To this end, we use the result of [28], where the $6 \mathrm{~d} \operatorname{Sp}(1)$ gauge theory with 10 flavors and a tensor is computed. The elliptic genus for one-string $\tilde{Z}_{(1)}$ and that for two strings $\tilde{Z}_{(2)}$ are given by $[28,29,38]$,

$$
\begin{aligned}
\tilde{Z}_{(1)}= & \frac{1}{2} \frac{\eta^{2}}{\theta_{1}^{2}(q)} \sum_{I=1}^{4} \frac{\eta^{2}}{\theta_{I}(\tilde{A}) \theta_{I}\left(\tilde{A}^{-1}\right)} \prod_{l=1}^{10} \frac{\theta_{I}\left(\tilde{y}_{l}\right)}{\eta} \\
\tilde{Z}_{(2)}= & \frac{\eta^{8}}{\theta_{1}^{2}(q) \theta_{1}^{2}\left(\tilde{A}^{2} q\right) \theta_{1}^{2}\left(\tilde{A}^{2} q^{-1}\right) \theta_{1}^{2}\left(\tilde{A}^{2}\right)} \prod_{l=1}^{10} \frac{\theta_{1}\left(\tilde{y}_{l} \tilde{A}\right) \theta_{1}\left(\tilde{y}_{l} \tilde{A}^{-1}\right)}{\eta^{2}} \\
& +\frac{1}{2} \frac{\eta^{4}}{\theta_{1}^{2}(q) \theta_{1}^{2}\left(q^{2}\right)} \sum_{I=1}^{4} \frac{\eta^{4}}{\theta_{I}^{2}\left(\tilde{A} q^{1 / 2}\right) \theta_{I}^{2}\left(\tilde{A} q^{-1 / 2}\right)} \prod_{l=1}^{10} \frac{\theta_{I}\left(\tilde{y}_{l} q^{1 / 2}\right) \theta_{I}\left(\tilde{y}_{l} q^{-1 / 2}\right)}{\eta^{2}} \\
& +\frac{1}{4} \frac{\eta^{4}}{\theta_{1}^{4}(q)} \sum_{(I, J, K) \in S} \frac{(-1)^{\delta_{K}, 1} \eta^{4} \theta_{I}^{2}(1)}{\theta_{I}^{2}(q) \theta_{J}^{2}(\tilde{A}) \theta_{K}^{2}(\tilde{A})} \prod_{l=1}^{10} \frac{\theta_{J}\left(\tilde{y}_{l}\right) \theta_{K}\left(\tilde{y}_{l}\right)}{\eta^{2}},
\end{aligned}
$$

where in the last line, $S=\{(2,2,1),(3,3,1),(4,4,1),(2,3,4),(3,4,2),(4,2,3)\}$ and $\delta_{I, J}$ is the Kronecker delta. The $\tilde{y}_{i}(i=1, \cdots, 10), \tilde{A}, \phi$, and $\tilde{\mathfrak{q}}$ denote the fugacities for an $\operatorname{SO}(20)$ flavor symmetry, $\operatorname{Sp}(1)$ gauge symmetry, counting the number of the self-dual strings, and instanton factor, respectively. The explicit forms of the Jacobi's theta functions $\theta_{I}(x)$ are given in appendix A. The partition function of the $6 \mathrm{~d} \mathrm{Sp}(1)$ gauge theory then takes the form

$$
\tilde{Z}^{6 \mathrm{~d} \operatorname{Sp}(1)}=\operatorname{PE}\left[\tilde{F}_{(0)}+\tilde{F}_{(1)} \phi+\tilde{F}_{(2)} \phi^{2}+\mathcal{O}\left(\phi^{3}\right)\right]
$$

where

$$
\tilde{F}_{(1)}=\tilde{Z}_{(1)}, \quad \tilde{F}_{(2)}=\tilde{Z}_{(2)}-\frac{1}{2} \tilde{Z}_{(1)}^{2}-\frac{1}{2} \tilde{Z}_{(1)}\left(* \rightarrow *^{2}\right) .
$$

The overall factor $\tilde{F}_{(0)}$ comes from "zero string" contribution explained in [29],

$$
\tilde{F}_{(0)}=\frac{q}{(1-q)^{2}}\left(2\left(\tilde{A}^{2}+\tilde{A}^{-2}\right)-\left(\tilde{A}+\tilde{A}^{-1}\right) \sum_{i=1}^{10}\left(\tilde{y}_{i}+\tilde{y}_{i}^{-1}\right)\right)\left(\frac{\tilde{\mathfrak{q}}}{1-\tilde{\mathfrak{q}}}+\frac{1}{2}\right) \text {. }
$$

We summarize the duality map between two theories among the $6 \mathrm{~d} \operatorname{Sp}(1)$ gauge theory with 10 flavors and a tensor $(6 \mathrm{~d} \operatorname{Sp}(1)+10 \mathbf{F}+1 \mathbf{T}), 5 \mathrm{~d} \operatorname{Sp}(2)$ gauge theory with 10 flavors $(5 \mathrm{~d} \mathrm{Sp}(2)+10 \mathbf{F})$, and $5 \mathrm{~d} \mathrm{SU}(3)_{0}$ gauge theory with 10 flavors $\left(5 \mathrm{~d} \mathrm{SU}(3)_{0}+10 \mathbf{F}\right)$ : 
- The duality map between $6 \mathrm{~d} \operatorname{Sp}(1)+10 \mathbf{F}+1 \mathbf{T}$ and $5 \mathrm{~d} \operatorname{Sp}(2)+10 \mathbf{F}$ :

$$
\begin{aligned}
& \tilde{y}_{i}=y_{i}^{\prime-1}(i=1, \ldots, 5), \quad \tilde{y}_{i}=y_{i}^{\prime}(i=6, \ldots, 9), \quad \tilde{y}_{10}=\mathfrak{q}^{\prime-2} y_{10}^{\prime}, \\
& \phi=\mathfrak{q}^{\prime} y_{10}^{\prime-1} A_{1}^{\prime}, \quad \tilde{A}=A_{2}^{\prime}, \quad \tilde{\mathfrak{q}}=\mathfrak{q}^{\prime 2},
\end{aligned}
$$

where $y_{i}^{\prime}(i=1, \cdots, 10), A_{j}^{\prime}(j=1,2), \mathfrak{q}^{\prime}$ are the flavor fugacities, the Coulomb branch moduli, and instanton factor, respectively.

- The duality map between $6 \mathrm{~d} \operatorname{Sp}(1)+10 \mathbf{F}+1 \mathbf{T}$ and $5 \mathrm{~d} \mathrm{SU}(3)_{0}+10 \mathbf{F}$, which comes from (3.31) and (2.14) with $\mathfrak{q}^{\prime}=\mathfrak{q}$ :

$$
\begin{aligned}
\tilde{y}_{i} & =\mathfrak{q}^{-1 / 2} \Lambda_{\mathrm{SU}(3)}^{-1} M_{i}^{-1}(i=1, \ldots, 5), & \tilde{y}_{i} & =\mathfrak{q}^{1 / 2} \Lambda_{\mathrm{SU}(3)} M_{i}(i=6, \ldots, 9), \\
\tilde{y}_{10} & =\mathfrak{q}^{-3 / 2} \Lambda_{\mathrm{SU}(3)} M_{10}, & & \phi=\mathfrak{q}^{2} M_{10}^{-1} A_{1}, \\
\tilde{A} & =\mathfrak{q}^{1 / 2} \Lambda_{\mathrm{SU}(3)} A_{2}, & \tilde{\mathfrak{q}} & =\mathfrak{q}^{2} .
\end{aligned}
$$

Using the duality map above, one can readily check that two partition functions do agree with each other by a double expanding in terms of the 5 d Coulomb parameter $A_{1}$ and the instanton fugacity $\mathfrak{q}[29]$.

Some comments are in order. First, this transformation is slightly different from the one given in [29], however, the difference is just the convention, and our convention is more useful to consider the flavor decoupling limit that we will discuss in appendix D, so that we adopt them. Second, we check the agreement of the partition function and the elliptic genus by the double expansion as a power series of $A_{1}$ and $\mathfrak{q}$ up to second order through the duality map (3.32).

The usual Higgsing from $6 \mathrm{~d} \operatorname{Sp}(1)$ gauge theory with 10 flavors and a tensor, to $6 \mathrm{~d}$ E-string theory, is achieved by setting the Coulomb branch parameter $\tilde{A}$ and two mass parameters $\tilde{y}_{3}, \tilde{y}_{8}$ as

$$
\tilde{A}=\tilde{y}_{3}^{-1}=\tilde{y}_{8},
$$

which is equivalent to (3.9) through the map (3.31). Then the elliptic genera of one and two strings become

$$
\begin{aligned}
\tilde{Z}_{(1)} \rightarrow & \frac{1}{2} \frac{\eta^{2}}{\theta_{1}^{2}(q)} \sum_{I=1}^{4} \prod_{l \in \mathcal{I}} \frac{\theta_{I}\left(\tilde{y}_{l}\right)}{\eta}, \\
\tilde{Z}_{(2)} \rightarrow & \frac{1}{2} \frac{\eta^{4}}{\theta_{1}^{2}(q) \theta_{1}^{2}\left(q^{2}\right)} \sum_{I=1}^{4} \prod_{l \in \mathcal{I}} \frac{\theta_{I}\left(\tilde{y}_{l} q^{1 / 2}\right) \theta_{I}\left(\tilde{y}_{l} q^{-1 / 2}\right)}{\eta^{2}} \\
& +\frac{1}{4} \frac{\eta^{4}}{\theta_{1}^{4}(q)} \sum_{(I, J, K) \in S} \frac{\theta_{I}^{2}(1)}{\theta_{I}^{2}(q)} \prod_{l \in \mathcal{I}} \frac{\theta_{J}\left(\tilde{y}_{l}\right) \theta_{K}\left(\tilde{y}_{l}\right)}{\eta^{2}},
\end{aligned}
$$

which agree with those for the E-string theory up to redefinitions of the parameters. For the elliptic genus of one string, the summation of the product of the theta functions can be expressed as $E_{8}$ theta function which is defined as the summation over the $E_{8}$ root lattice $\Gamma_{8}$,

$$
\sum_{I=1}^{4} \prod_{l \in \mathcal{I}} \frac{\theta_{I}\left(\tilde{y}_{l}\right)}{\eta}=2 \sum_{\vec{w} \in \Gamma_{8}} \exp [\pi \mathrm{i} \tau \vec{w}+2 \pi \mathrm{i} \vec{\mu} \cdot \vec{w}],
$$


where $\vec{w}=\left(w_{1}, \cdots, w_{8}\right), \vec{\mu}=\left(\mu_{1}, \cdots, \mu_{8}\right)$, and $\mu_{i}=\frac{1}{2 \pi \mathrm{i}} \log y_{i}, \tau=\frac{1}{2 \pi \mathrm{i}} \log \mathfrak{q}$. One can also express the elliptic genus of two strings as combinations of the $E_{8}$ theta functions, hence the E-strings enjoy $E_{8}$ Weyl symmetry.

Now we implement the defect Higgsing. In a similar fashion, we set

$$
\tilde{A}=q^{-M} \tilde{y}_{3}^{-1}=\tilde{y}_{8},
$$

which is consistent with (3.15) with the duality map (3.32). The resulting elliptic genera of the E-strings with the defect are given by

$$
\begin{aligned}
\tilde{Z}_{(1)}^{\text {defect }}= & \frac{1}{2} \frac{\eta^{2}}{\theta_{1}^{2}(q)} \sum_{I=1}^{4}\left(\prod_{l \in \mathcal{I}} \frac{\theta_{I}\left(\tilde{y}_{l}\right)}{\eta}\right)\left(\frac{\theta_{I}(\tilde{z})}{\theta_{I}\left(q^{M} \tilde{z}\right)}\right), \\
\tilde{Z}_{(2)}^{\text {defect }}= & \frac{1}{2} \frac{\eta^{4}}{\theta_{1}^{2}(q) \theta_{1}^{2}\left(q^{2}\right)} \sum_{I=1}^{4}\left(\prod_{l \in \mathcal{I}} \frac{\theta_{I}\left(\tilde{y}_{l} q^{1 / 2}\right) \theta_{I}\left(\tilde{y}_{l} q^{-1 / 2}\right)}{\eta^{2}}\right)\left(\frac{\theta_{I}\left(\tilde{z} q^{1 / 2}\right) \theta_{I}\left(\tilde{z} q^{-1 / 2}\right)}{\theta_{I}\left(q^{M+1 / 2} \tilde{z}\right) \theta_{I}\left(q^{M-1 / 2} \tilde{z}\right)}\right) \\
& +\frac{1}{4} \frac{\eta^{4}}{\theta_{1}^{4}(q)} \sum_{(I, J, K) \in S} \frac{\theta_{I}^{2}(1)}{\theta_{I}^{2}(q)} \prod_{l \in \mathcal{I}} \frac{\theta_{J}\left(\tilde{y}_{l}\right) \theta_{K}\left(\tilde{y}_{l}\right)}{\eta^{2}}\left(\frac{\theta_{J}(\tilde{z}) \theta_{K}(\tilde{z})}{\theta_{J}\left(q^{M} \tilde{z}\right) \theta_{K}\left(q^{M} \tilde{z}\right)}\right)
\end{aligned}
$$

where $\tilde{z}=\tilde{y}_{3}$ is the position of the defect insertion.

We note here that unlike the usual Higgsing, $\tilde{Z}_{(1)}^{\text {Defect }}$ and $\tilde{Z}_{(2)}^{\text {Defect }}$ now are not invariant under $\mathrm{E}_{8}$ Weyl symmetry, rather they are invariant under the $\mathrm{SO}(16)$ Weyl symmetry,

$$
\tilde{y}_{i} \leftrightarrow \tilde{y}_{j}, \quad \tilde{y}_{i} \leftrightarrow \tilde{y}_{j}^{-1}
$$

This implies that the $\mathrm{E}_{8}$ global symmetry of the elliptic genus for the E-string is broken $\mathrm{SO}(16)$ due to the presence of the defects.

To see the symmetry breaking more explicitly, let us consider the defect Higgsing of the elliptic genus expressed by the characters given in [38]. A part of the contributions to the elliptic genus of one string $\tilde{Z}_{(1)}$ can be expressed as the character,

$$
\tilde{Z}_{(1)}=\frac{1}{(1-q)\left(1-q^{-1}\right)} \frac{\tilde{\mathfrak{q}}^{1 / 2}}{\left(1-\tilde{A}^{2}\right)\left(1-\tilde{A}^{-2}\right)}\left(\tilde{Z}_{(1)}^{\mathrm{SO}, \mathrm{SU}}+\tilde{Z}_{(1)}^{\mathrm{SU}}\right)+\mathcal{O}\left(\tilde{\mathfrak{q}}^{3 / 2}\right)
$$

where we decompose $\tilde{Z}_{(1)}$ into two parts: the contributions involving $\mathrm{SO}(20)$ characters and those not depending on $\mathrm{SO}(20)$ characters,

$$
\begin{aligned}
\tilde{Z}_{(1)}^{\mathrm{SO}, \mathrm{SU}}= & -\chi_{\overline{\mathbf{5 1 2}}}^{\mathrm{SO}(20)} \chi_{1 / 2}^{\mathrm{SU}(2)}(\tilde{A})+2 \chi_{\mathbf{5 1 2}}^{\mathrm{SO}(20)}+2 \chi_{\mathbf{2 0}}^{\mathrm{SO}(20)} \chi_{3 / 2}^{\mathrm{SU}(2)}(\tilde{A})-4 \chi_{\mathbf{2 0}}^{\mathrm{SO}(20)} \chi_{1 / 2}^{\mathrm{SU}(2)}(\tilde{A}) \\
& -\chi_{\mathbf{1 9 0}}^{\mathrm{SO}(20)} \chi_{1}^{\mathrm{SU}(2)}(\tilde{A})+3 \chi_{\mathbf{1 9 0}}^{\mathrm{SO}(20)} \\
\tilde{Z}_{(1)}^{\mathrm{SU}}= & 6 \chi_{1 / 2}^{\mathrm{SU}(2)}(q)-2 \chi_{1}^{\mathrm{SU}(2)}(\tilde{A}) \chi_{1 / 2}^{\mathrm{SU}(2)}(q)-5 \chi_{1}^{\mathrm{SU}(2)}(\tilde{A})-3 \chi_{2}^{\mathrm{SU}(2)}(\tilde{A})
\end{aligned}
$$


where relevant the $\mathrm{SO}(20)$ characters are given by

$$
\begin{aligned}
\chi_{\mathbf{5 1 2}}^{\mathrm{SO}(20)} & =\frac{1}{2}\left(\prod_{i=1}^{10}\left(\tilde{y}_{i}^{1 / 2}+\tilde{y}_{i}^{-1 / 2}\right)+\prod_{i=1}^{10}\left(\tilde{y}_{i}^{1 / 2}-\tilde{y}_{i}^{-1 / 2}\right)\right), \\
\chi_{\overline{\mathbf{5 1 2}}}^{\mathrm{SO}(20)} & =\frac{1}{2}\left(\prod_{i=1}^{10}\left(\tilde{y}_{i}^{1 / 2}+\tilde{y}_{i}^{-1 / 2}\right)-\prod_{i=1}^{10}\left(\tilde{y}_{i}^{1 / 2}-\tilde{y}_{i}^{-1 / 2}\right)\right), \\
\chi_{\mathbf{1 9 0}}^{\mathrm{SO}(20)} & =\sum_{1 \leq i<j \leq 10}\left(\tilde{y}_{i}+\tilde{y}_{i}^{-1}\right)\left(\tilde{y}_{j}+\tilde{y}_{j}^{-1}\right)+10, \\
\chi_{\mathbf{2 0}}^{\mathrm{SO}(20)} & =\sum_{i=1}^{10}\left(\tilde{y}_{i}+\tilde{y}_{i}^{-1}\right),
\end{aligned}
$$

as well as the $\mathrm{SU}(2)$ character,

$$
\chi_{n}^{\mathrm{SU}(2)}(x)=\frac{x^{2 n+1}-x^{-2 n-1}}{x-x^{-1}} .
$$

By the defect Higgsing (3.36), the part of the elliptic genus of one string (3.40a) reduces to

$$
\begin{aligned}
\tilde{Z}_{(1)}^{\mathrm{SOSU}} \rightarrow( & -\left(q^{M} \tilde{z}-q^{-M} \tilde{z}^{-1}\right)\left(q^{M / 2} \tilde{z}-q^{-M / 2} \tilde{z}^{-1}\right) \chi_{\mathbf{1 2 8}}^{\mathrm{SO}(16)} \\
& -\left(q^{M} \tilde{z}-q^{-M} \tilde{z}^{-1}\right)\left(q^{M / 2}-q^{-M / 2}\right) \chi_{\mathbf{1 2 8}}^{\mathrm{SO}(16)}-\left(q^{M} \tilde{z}-q^{-M} \tilde{z}^{-1}\right)^{2} \chi_{\mathbf{1 2 0}}^{\mathrm{SO}(16)} \\
& \left.+\left(q^{M} \tilde{z}-q^{-M} \tilde{z}^{-1}\right)^{2}\left(q^{M / 2} \tilde{z}-q^{-M / 2} z^{-1}\right)\left(q^{M / 2}-q^{-M / 2}\right) \chi_{\mathbf{1 6}}^{\mathrm{SO}(16)}\right),
\end{aligned}
$$

where we further introduce the $\mathrm{SO}(16)$ characters,

$$
\begin{aligned}
\chi_{\mathbf{1 2 8}}^{\mathrm{SO}(16)} & =\frac{1}{2}\left(\prod_{i \in \mathcal{I}}\left(\tilde{y}_{i}^{1 / 2}+\tilde{y}_{i}^{-1 / 2}\right)+\prod_{i \in \mathcal{I}}\left(\tilde{y}_{i}^{1 / 2}-\tilde{y}_{i}^{-1 / 2}\right)\right), \\
\chi_{\mathbf{1 2 8}}^{\mathrm{SO}(16)} & =\frac{1}{2}\left(\prod_{i \in \mathcal{I}}\left(\tilde{y}_{i}^{1 / 2}+\tilde{y}_{i}^{-1 / 2}\right)-\prod_{i \in \mathcal{I}}\left(\tilde{y}_{i}^{1 / 2}-\tilde{y}_{i}^{-1 / 2}\right)\right), \\
\chi_{\mathbf{1 2 0}}^{\mathrm{SO}(16)} & =\sum_{i, j \in \mathcal{I}, i<j}\left(\tilde{y}_{i}+\tilde{y}_{i}^{-1}\right)\left(\tilde{y}_{j}+\tilde{y}_{j}^{-1}\right), \\
\chi_{\mathbf{1 6}}^{\mathrm{SO}(16)} & =\sum_{i \in \mathcal{I}}\left(\tilde{y}_{i}+\tilde{y}_{i}^{-1}\right) .
\end{aligned}
$$

When we set $M=0$ corresponding to the Higgsing, we can combine $\chi_{\mathbf{1 2 8}}^{\mathrm{SO}(16)}$ and $\chi_{\mathbf{1 2 0}}^{\mathrm{SO}(16)}$ into $\mathrm{E}_{8}$ character,

$$
-\left(\tilde{z}-\tilde{z}^{-1}\right)^{2}\left(\chi_{\mathbf{1 2 8}}^{\mathrm{SO}(16)}+\chi_{\mathbf{1 2 0}}^{\mathrm{SO}(16)}\right)=-\left(\tilde{z}-\tilde{z}^{-1}\right)^{2} \chi_{\mathbf{2 4 8}}^{\mathrm{E}_{8}},
$$

which is nothing but the symmetry of E-string, Weyl symmetry of $E_{8}$. In order to enhance the symmetry, the coefficients of $\chi_{\mathbf{1 2 8}}^{\mathrm{SO}(16)}$ and $\chi_{\mathbf{1 2 0}}^{\mathrm{SO}(16)}$ in (3.43) have to be the same, whereas those of $\chi_{\frac{\mathrm{SO}(16)}{\mathbf{1 2 8}}}$ and $\chi_{\mathbf{1 6}}^{\mathrm{SO}(16)}$ have to vanish, however, there is no such solution except for trivial cases, $M=0$. Therefore, we conclude that the symmetry of E-string is broken to $\mathrm{SO}(16)$ by introducing the defect. 


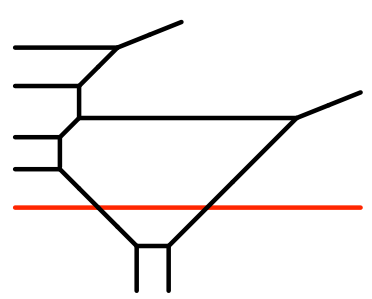

$(a)$

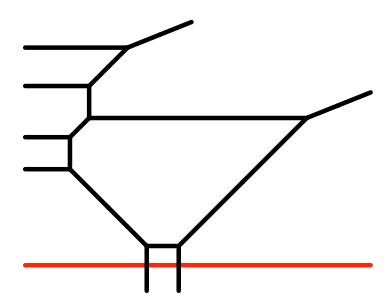

(b)

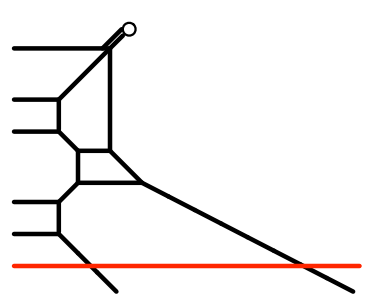

(c)

Figure 8. Different unHiggsed 5-brane configurations for $\mathrm{SU}(2)$ gauge theory with 4 flavors and defects, associated with different global symmetries: (a) $\mathrm{SU}(4) \times \mathrm{SU}(2) \times \mathrm{SU}(2)$, (b) $\mathrm{SU}(4) \times \mathrm{U}(1) \times \mathrm{SU}(2)$, and (c) $\mathrm{SU}(5) \times \mathrm{U}(1)$. The red lines are D5-branes where the defect Higgsing is applied. A white dot is 7-brane.

\section{Global symmetry}

In this section, we comment on global symmetry in the presence of codimension 2 defects. In computing the defect partition function, we used geometric transitions, where we construct "unHiggsed" 5-brane configuration and perform the defect Higgsing. As unHiggsed theory does not have any defect attached, the defects are introduced through defect Higgsing. Theories with defects that can be geometrically engineered hence captures some subalgebra of global symmetry of the unHiggsed theory. For instance, the partition function for the E-string theory with defects that we have computed in the previous section show manifest $\mathrm{SO}(16)$ global symmetry. We obtained it through the defect Higgsing of $6 \mathrm{~d} \mathrm{Sp}(1)$ gauge theory with 10 flavors which has $\mathrm{SO}(20)$ global symmetry. The defect of the E-string that we considered thus naturally has an $\mathrm{SO}(16)$ which can be understood as $\mathrm{SO}(20)$ global symmetry of the unHiggsed theory is broken to $\mathrm{SO}(16)$ along with the defect Higgsing.

In M-theory perspective, $E_{8}$ global symmetry of E-string is the symmetry that arises as E-string probes M9 brane. Defects on E-string are expected not to disturb M9 brane, and hence one may expect to still see an $E_{8}$ symmetry for E-string theory with defects. Global symmetry of defect partition function, however, may depend on how we compute the defects. For instance, we can introduce different codimension 2 defects via geometric transitions for a given 5d theory by considering defect Higgsings on different unHiggsed 5-brane webs. These different defect Higgsings may capture different global symmetry, which could be the perturbative symmetry or more than the perturbative symmetry. As an instructive example, let us consider $\mathrm{SU}(2)$ theory with 4 flavors, whose perturbative symmetry is $\mathrm{SO}(8) \times \mathrm{U}(1)$. In figure 8 , three different unHiggsed 5-brane configurations are considered and each gives rise to different manifest global symmetries as depicted. The red lines in figure 8 are D5-branes which we perform the defect Higgsing. The 5-brane configurations (a) and (b) are, in particular, instructive. Both unHiggsed 5-brane webs are the same as $\mathrm{SU}(3)_{0}$ gauge theory with 6 flavors, their resulting global symmetries however are different. Global symmetry for (a) is $\mathrm{SU}(4) \times \mathrm{SU}(2) \times \mathrm{SU}(2)$, while that for (b) is $\mathrm{SU}(4) \times \mathrm{U}(1) \times \mathrm{SU}(2)$. Here $\mathrm{SU}(4)$ is the symmetry coming from the interchange of four flavor masses, and $\mathrm{SU}(2)$ arises from two parallel $(2,1) 5$-branes in figure 8 (a) and (b), 
which is associated with instanton. Now the difference can be explained as follows. In (a), the defect Higgsing is applied to the internal D5-brane, which leaves two parallel $(0,1)$ 5 -branes on the bottom symmetric, accounting for the $\mathrm{SU}(2)$ part of its global symmetry. In (b), on the other hand, the defect Higgsings is applied to an outer D5-brane which makes two parallel $(0,1) 5$-branes on the bottom distinguishable, hence breaking $\mathrm{SU}(2)$ into U(1). The unHiggsed 5-brane configuration (c) is also noticeable. The resulting global symmetry is $\mathrm{SU}(5) \times \mathrm{U}(1)$, which is obtained from an $\mathrm{SU}(4)_{-\frac{1}{2}}$ gauge theory with 7 flavors, with usual Higgsing in the upper part and also with a defect Higgsing in the lower part. From these three examples, it is clear that global symmetry of defect Higgsings comes from the global symmetry structure of unHiggsed 5-brane web, which could be simply perturbative symmetry or one of the maximal compact subgroup of the enhanced global symmetry, $\mathrm{SO}(10)$ in this particular example.

As there could be many more unHiggsed 5-brane configurations that one can geometrically engineer, we expect different global symmetry depending on unHiggsed 5-brane that we used for the computations.

\section{Conclusion}

In this paper, we computed the defect partition function of E-string theory on a circle, from 5-brane webs by applying the defect Higgsing, based on two 5-brane configurations: one with two O5-planes and the other without O5-planes. Though two 5-brane configurations look different, both parameter phases actually describe $5 \mathrm{~d} \mathrm{SU}(3)_{0}$ gauge theory with 10 flavors, as the 5-brane web with two O5-planes is deformed to describe $\mathrm{SU}(3)$ gauge theory phases. We however referred to the one with O5-planes as $\mathrm{Sp}(2)$ gauge theory with 10 flavors just to distinguish natural 5-brane web, without orientifolds, for SU(3) gauge theory with 10 flavors. As 6d E-string theory on a circle is realized as $5 \mathrm{~d} \operatorname{Sp}(1)$ gauge theory with 8 flavors, we applied a suitable defect Higgsing on these 5-brane webs to obtain the surface defect partition function for $5 \mathrm{~d} \mathrm{Sp}(1)$ gauge theory with 8 flavors. The resulting partition function has the defect modulus which corresponds to the position on 5-brane where the defect is inserted. With the defect modulus, the partition function can be understood as the open topological string partition function. We compared our defect partition function with $6 \mathrm{~d}$ elliptic genus result where the same defect Higgsing is implemented. We carried out our computation of the defect partition function up to 2-instantons and confirmed that the results agree up to that order.

For the 5-brane configurations that we considered in the paper, the insertion of the defect breaks global symmetry from $E_{8}$ to $\mathrm{SO}(16)$. This can be explicitly seen from the defect partition function obtained from the elliptic genus where the theta function at a given instanton order is invariant under the $\mathrm{SO}(16)$ Weyl transformation rather than the $E_{8}$ transformation. From the partition function of $5 \mathrm{~d} \operatorname{Sp}(1)$ gauge theory with 8 flavors, the $\mathrm{SO}(16)$ corresponds to the perturbative $\mathrm{SO}(16)$ flavor symmetry. The corresponding 5-brane web suggests that flavors are not affected by the defect and hence 8 flavor branes can be put closer to one of O5-planes so that they enjoy an $\mathrm{SO}(16)$ symmetry. In this way, one can see that regardless of the number of the defects, $\mathrm{SO}(16)$ global symmetry remains. 
Together with $\mathrm{U}(1)$ coming from the KK modes, the defect partition function hence has $\mathrm{SO}(16) \times \mathrm{U}(1)_{K K}$ symmetry.

We note that there are different ways of realizing $5 \mathrm{~d}$ brane system with defects, as discussed in section 4. Depending on how we implement the defect Higgsing, we may see different global symmetry structures. It is however that such apparent global symmetries are in fact a subgroup of the enhanced global symmetry, as one can readily restore the enhanced global symmetry when setting the number of defects to zero. Even for the case of the E-string theory with defects, global symmetry may restore to be $E_{8}$ if there is a nontrivial unHiggsed 5-brane configuration or nontrivial reparameterization involving the defect modulus such that it respects $E_{8}$ Weyl transformation. It is hence interesting to further study how the global symmetry structure is broken or hidden in the presence of defects. For instance, quantization of SW curve or associated integrable system [9, 40-42].

Some defects can be taken away by taking the defect insertion to infinity. In our setup, however, as we inserted the defect in between two NS5 branes, it is not possible to decouple the defects. Instead, one can set the number of the defects to zero, then one naturally recovers the E-string partition on a circle which restores $E_{8}$ global symmetry.

It is straightforward to generalize our computation to higher rank gauge theories. For instance, $6 \mathrm{~d} \mathcal{N}=1 \mathrm{Sp}(N)$ gauge theory with $2 N+8$ flavors with a tensor compactified on a circle can be realized as a 5-brane web with two O5-planes describing $5 \mathrm{~d} \mathcal{N}=1 \mathrm{Sp}(N+1)$ gauge theory with $2 N+8$ flavors. As we can apply the defect Higgsing to it, we expect to obtain $5 \mathrm{~d} \operatorname{Sp}(N)$ gauge theory with $2 N+6$ flavors and defects. Global symmetry for the theory with defects would be an $\mathrm{SO}(4 N+12) \times \mathrm{U}(1)_{K K}$, as the corresponding 5-brane configuration suggests that there are O5-planes with $2 N+6$ flavor D7-branes, and the instanton gives a $\mathrm{U}(1)$.

We can also consider the defect Higgsing for the system with fewer flavors. Starting from a $5 \mathrm{~d}$ KK theory with defects, say $5 \mathrm{~d} \mathcal{N}=1 \mathrm{Sp}(N+1)$ gauge theory with $2 N+8$ flavors and defects, one can decouple flavors one by one to get $5 \mathrm{~d} \mathcal{N}=1 \operatorname{Sp}(N+1)$ gauge theory with $N_{f}<2 N+8$ flavors and defects. For example, we worked out explicitly the partition function for $5 \mathrm{~d} \mathrm{Sp}(2)$ gauge theory with 9 and 8 flavors in appendix D.

\section{Acknowledgments}

We thank Joonho Kim, Hee-Cheol Kim, and Kimyeong Lee for useful discussions. SSK thanks Interdisciplinary Center for Theoretical Study, University of Science and Technology of China for hospitality, KIAS and POSTECH for his visit where part of work is done, and also thanks APCTP for hosting the Focus program "Strings, Branes and Gauge Theories." YS thanks University of Electronic Science and Technology of China and South West Jiaotong University for hospitality for his visit. YS is supported by a grant from the NSF of China with Grant No: 11675167 and 11947301. FY is supported by the NSFC grant No. 11950410490, the Fundamental Research Funds for the Central Universities A0920502051904-48, by Startup research grant A1920502051907-2-046, in part by NSFC grant No. 11501470 and No. 11671328, and by Recruiting Foreign Experts Program No. T2018050 granted by SAFEA. 


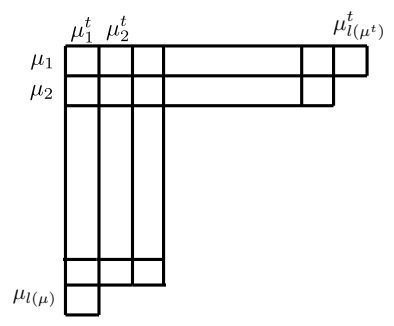

Figure 9. The Young diagram and its parameters.

\section{A Conventions and notations}

\section{A.1 Topological vertex}

Here we provide some definition and notation we use in this paper. We first define the Young diagram as in figure 9 . The variables $\mu_{i}, \mu_{j}^{t}, l(\mu)$ denote the number of boxes in the $i$-th row, the number of boxes in the $j$-th column, and the number of the columns. We also define the following quantities,

$$
|\mu|=\sum_{i}^{l(\mu)} \mu_{i}, \quad\|\mu\|^{2}=\sum_{i}^{l(\mu)} \mu_{i}^{2}, \quad\left\|\mu^{t}\right\|^{2}=\sum_{i}^{l(\mu)} \mu_{i}^{t^{2}} .
$$

Based on the notation of the Young diagram, we define the topological vertex $C_{\lambda \mu \nu}$ [23],

$$
C_{\lambda \mu \nu}=q^{\frac{\|\mu\|^{2}-\left\|\mu^{t}\right\|^{2}}{2}} s_{\nu^{t}}\left(q^{-\rho}\right) \sum_{\eta} s_{\lambda^{t} / \eta}\left(q^{-\nu-\rho}\right) s_{\mu / \eta}\left(q^{-\rho-\nu^{t}}\right)
$$

where $\lambda, \mu, \nu$ and $\eta$ are the Young diagrams, and $q$ relates to the string coupling, $q=\mathrm{e}^{\mathrm{i} g_{s}}$. The function $s_{\mu}(x)$ is Schur function defined by,

$$
s_{\mu}\left(x_{1}, \ldots, x_{N}\right)=\frac{\operatorname{det} x_{j}^{\mu_{i}+N-i}}{\operatorname{det} x_{j}^{N-i}},
$$

and $s_{\mu / \eta}(x)$ is skew Schur function defined by the summation of the Schur function with the weight $N_{\nu \eta}^{\mu}$ called as Littlewood-Richardson coefficients,

$$
s_{\mu / \eta}(x)=\sum_{\nu} N_{\nu \eta}^{\mu} s_{\nu}(x) .
$$

We also define the framing factor $f_{\mu}$,

$$
f_{\mu}=(-1)^{|\mu|} q^{-\frac{\left.\|\mu\|\right|^{2}-\| \mu^{t}||^{2}}{2}} .
$$

From these ingredients, the gluing rule for two vertices is given by (see also figure 11),

$$
\sum_{\mu} C_{\lambda_{1} \nu_{1} \mu} C_{\lambda_{2} \nu_{2} \mu^{t}}(-Q)^{|\mu|} f_{\mu}^{n}, \quad n=\left(p_{1}, q_{1}\right) \wedge\left(p_{2}, q_{2}\right) .
$$

By gluing some vertices, we can calculate a partition function of topological strings on non-compact toric Calabi-Yau manifolds. One can find more detailed computations and some examples in [23]. 


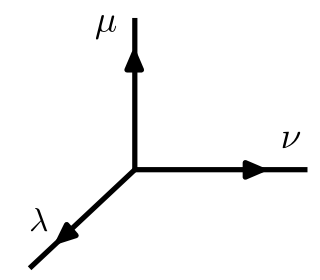

Figure 10. The pictorial description of the topological vertex.

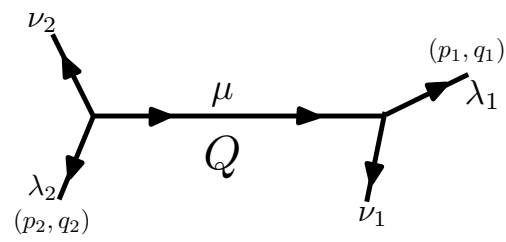

Figure 11. The gluing rule of two vertices.

\section{A.2 Theta function}

To provide the elliptic genus of E-strings, we provide the definition of Jacobi's theta function,

$$
\begin{aligned}
& \theta_{1}(p ; y):=-\mathrm{i} p^{1 / 8} y^{1 / 2} \prod_{n=1}^{\infty}\left(1-p^{n}\right)\left(1-p^{n} y\right)\left(1-p^{n-1} y^{-1}\right), \\
& \theta_{2}(p ; y)=p^{1 / 8} y^{1 / 2} \prod_{n=1}^{\infty}\left(1-p^{n}\right)\left(1+p^{n} y\right)\left(1+p^{n-1} y^{-1}\right), \\
& \theta_{3}(p ; y)=\prod_{n=1}^{\infty}\left(1-p^{n}\right)\left(1+p^{n-1 / 2} y\right)\left(1+p^{n-1 / 2} y^{-1}\right) \\
& \theta_{4}(p ; y)=\prod_{n=1}^{\infty}\left(1-p^{n}\right)\left(1-p^{n-1 / 2} y\right)\left(1-p^{n-1 / 2} y^{-1}\right)
\end{aligned}
$$

They satisfy the following reflection formula,

$$
\theta_{I}\left(p ; y^{-1}\right)=(-1)^{\delta_{I, 1}} \theta_{I}(p ; y)
$$

where $\delta_{I, j}$ is Kronecker delta, which is used to show that the elliptic genus of $6 \mathrm{~d} \operatorname{Sp}(1)$ gauge theory reduces to the one of E-strings after the Higgsing. In the section 3.3, we use short notation,

$$
\theta_{I}(\tilde{\mathfrak{q}} ; y)=\theta_{I}(y)
$$

where $\tilde{\mathfrak{q}}$ is instanton fugacity in $6 \mathrm{~d}$ theory relating to the one in $5 \mathrm{~d}$ theory $\mathfrak{q}, \tilde{\mathfrak{q}}=\mathfrak{q}^{2}$, as we denoted in (3.32). 


\section{A.3 Plethystic exponential}

To express the physical part of the partition functions, it is useful to express them as plethystic exponential defined by,

$$
\mathrm{PE}[f(\boldsymbol{x})]=\exp \left[\sum_{n=1}^{\infty} \frac{1}{n} f\left(\boldsymbol{x}^{n}\right)\right],
$$

where $\boldsymbol{x}=\left\{x_{1}, x_{2}, \ldots\right\}$ and $\boldsymbol{x}^{n}=\left\{x_{1}^{n}, x_{2}^{n}, \ldots\right\}$ For instance, an infinite product $\prod_{i, j=1}^{\infty}(1-$ $Q q^{i+j-1}$ ) can be expressed as

$$
\prod_{i, j=1}^{\infty}\left(1-Q q^{i+j-1}\right)=\exp \left[\sum_{n=1}^{\infty} \frac{1}{n} \frac{q^{n} Q^{n}}{\left(1-q^{n}\right)^{2}}\right]=\mathrm{PE}\left[\frac{q}{(1-q)^{2}} Q\right] .
$$

From this expression, the analytic continuation formula which can be understood as flop transition is given by

$$
\mathrm{PE}\left[\frac{q}{(1-q)^{2}} Q\right] \rightarrow \mathrm{PE}\left[\frac{q}{(1-q)^{2}} Q^{-1}\right]
$$

This formula is sometimes used to express the partition function as characters.

\section{B Kähler parameters of the Tao diagram}

Here, we summarize the expression for the Kähler parameters of the Tao diagram depicted in figure 1 in terms of the parameters of the $5 \mathrm{~d} \mathrm{SU}(3)$ gauge theory with 10 flavors. The derivation detail is given in the appendix of [29]. The Kähler parameters associated with the middle strip are given by

$$
\begin{aligned}
Q_{1} & =\frac{M_{1}}{A_{1}}, \quad \tilde{Q}_{1}=\frac{A_{1}}{M_{2}}, \quad Q_{2}=\frac{M_{2}}{A_{2}}, \quad \tilde{Q}_{2}=\frac{A_{2}}{M_{3}}, \\
Q_{3} & =\frac{M_{3}}{A_{3}}, \quad \tilde{Q}_{3}=\frac{A_{3}}{M_{5}}, \quad Q_{4}=\mathfrak{q} \sqrt{\frac{M_{4} M_{5}}{M_{1} M_{2} M_{3} M_{6} M_{7} M_{8} M_{9} M_{10}}}, \\
Q_{5} & =\frac{A_{3}}{M_{6}}, \quad \tilde{Q}_{5}=\frac{M_{7}}{A_{3}}, \quad Q_{6}=\frac{A_{2}}{M_{7}}, \quad \tilde{Q}_{6}=\frac{M_{8}}{A_{2}}, \\
Q_{7} & =\frac{A_{1}}{M_{8}}, \quad \tilde{Q}_{7}=\frac{M_{10}}{A_{1}}, \quad Q_{8}=\mathfrak{q} \sqrt{\frac{M_{6} M_{7} M_{8} M_{1} M_{2} M_{3} M_{4} M_{5}}{M_{9} M_{10}}}, \\
Q_{b_{1}} & =\mathfrak{q} A_{1} \sqrt{\frac{M_{2} M_{3} M_{4} M_{5} M_{6} M_{7} M_{8}}{M_{1} M_{9} M_{10}}}, \quad Q_{b_{2}}=\mathfrak{q} \frac{A_{1}}{A_{3}} \sqrt{\frac{M_{3} M_{4} M_{5} M_{6} M_{7}}{M_{1} M_{2} M_{8} M_{9} M_{10}}} \\
Q_{b_{3}} & =\frac{\mathfrak{q}}{A_{3}} \sqrt{\frac{M_{4} M_{5} M_{6}}{M_{1} M_{2} M_{3} M_{7} M_{8} M_{9} M_{10}}}
\end{aligned}
$$

whereas those for the spiral strip 1 and 2 are given by

$$
\begin{aligned}
& Q_{1}^{(1)}=\frac{1}{M_{2} M_{3} M_{4}}, \quad Q_{2}^{(1)}=\frac{M_{1}}{M_{5}}, \quad Q_{3}^{(1)}=\mathfrak{q} \sqrt{\frac{M_{1} M_{5}}{M_{2} M_{3} M_{4} M_{6} M_{7} M_{8} M_{9} M_{10}}}, \quad Q_{4}^{(1)}=\mathfrak{q} \sqrt{\frac{M_{1} M_{6} M_{7} M_{8} M_{9}}{M_{2} M_{3} M_{4} M_{5} M_{10}}}, \\
& Q_{5}^{(1)}=\mathfrak{q} \sqrt{\frac{M_{1} M_{10}}{M_{2} M_{3} M_{4} M_{5} M_{6} M_{7} M_{8} M_{9}}}, \quad Q_{6}^{(1)}=\mathfrak{q}^{2} \frac{M_{1}}{M_{10}}, \quad Q_{7}^{(1)}=\mathfrak{q}^{2} \frac{1}{M_{2} M_{3} M_{4}}, \quad Q_{8}^{(1)}=\mathfrak{q}^{2} \frac{M_{1}}{M_{5}},
\end{aligned}
$$




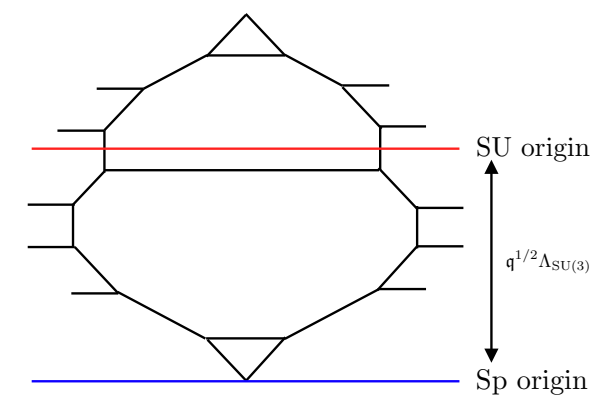

Figure 12. The difference of the SU origin and the Sp origin. In this figure we omit O5-plane.

$$
\begin{aligned}
& Q_{1}^{(2)}=\frac{1}{A_{1} M_{3} M_{4}}, \quad Q_{2}^{(2)}=\frac{M_{2}}{M_{5}}, \quad Q_{3}^{(2)}=\mathfrak{q} \sqrt{\frac{M_{2} M_{5}}{M_{1} M_{3} M_{4} M_{6} M_{7} M_{8} M_{9} M_{10}}}, \quad Q_{4}^{(2)}=\mathfrak{q} \sqrt{\frac{M_{2} M_{6} M_{7} M_{8} M_{9}}{M_{1} M_{3} M_{4} M_{5} M_{10}}}, \\
& Q_{5}^{(2)}=\mathfrak{q} \sqrt{\frac{M_{2} M_{10}}{M_{1} M_{3} M_{4} M_{5} M_{6} M_{7} M_{8} M_{9}}}, \quad Q_{6}^{(2)}=\mathfrak{q}^{2} \frac{M_{2}}{M_{10}}, \quad Q_{7}^{(2)}=\mathfrak{q}^{2} \frac{1}{M_{1} M_{3} M_{4}}, \quad Q_{8}^{(2)}=\mathfrak{q}^{2} \frac{M_{2}}{M_{5}}, \\
& Q_{1}^{(3)}=\frac{A_{3}}{M_{4}}, \quad Q_{2}^{(3)}=\frac{M_{3}}{M_{5}}, \quad Q_{3}^{(3)}=\mathfrak{q} \sqrt{\frac{M_{3} M_{5}}{M_{1} M_{2} M_{4} M_{6} M_{7} M_{8} M_{9} M_{10}}}, \quad Q_{4}^{(3)}=\mathfrak{q} \sqrt{\frac{M_{3} M_{6} M_{7} M_{8} M_{9}}{M_{1} M_{2} M_{4} M_{5} M_{10}}}, \\
& Q_{5}^{(3)}=\mathfrak{q} \sqrt{\frac{M_{3} M_{10}}{M_{1} M_{2} M_{4} M_{5} M_{6} M_{7} M_{8} M_{9}}}, \quad Q_{6}^{(3)}=\mathfrak{q}^{2} \frac{M_{3}}{M_{10}}, \quad Q_{7}^{(3)}=\mathfrak{q}^{2} \frac{1}{M_{1} M_{2} M_{4}}, \quad Q_{8}^{(3)}=\mathfrak{q}^{2} \frac{M_{3}}{M_{5}}, \\
& Q_{3}^{(4)}=\mathfrak{q} \sqrt{\frac{M_{4} M_{5}}{M_{1} M_{2} M_{3} M_{6} M_{7} M_{8} M_{9} M_{10}}}, \quad Q_{4}^{(4)}=\mathfrak{q} \sqrt{\frac{M_{4} M_{6} M_{7} M_{8} M_{9}}{M_{1} M_{2} M_{3} M_{5} M_{10}}}, \\
& Q_{5}^{(4)}=\mathfrak{q} \sqrt{\frac{M_{4} M_{10}}{M_{1} M_{2} M_{3} M_{5} M_{6} M_{7} M_{8} M_{9}}}, \quad Q_{6}^{(4)}=\mathfrak{q}^{2} \frac{M_{4}}{M_{10}}, \quad Q_{7}^{(4)}=\mathfrak{q}^{2} \frac{1}{M_{1} M_{2} M_{3}}, \quad Q_{8}^{(4)}=\mathfrak{q}^{2} \frac{M_{4}}{M_{5}}, \\
& Q_{1}^{(5)}=M_{7} M_{8} M_{9}, \quad Q_{2}^{(5)}=\frac{M_{10}}{M_{6}}, \quad Q_{3}^{(5)}=\mathfrak{q} \sqrt{\frac{M_{1} M_{2} M_{3} M_{4} M_{5} M_{7} M_{8} M_{9}}{M_{6} M_{10}}}, \quad Q_{4}^{(5)}=\mathfrak{q} \sqrt{\frac{M_{5} M_{7} M_{8} M_{9} M_{10}}{M_{1} M_{2} M_{3} M_{4} M_{6}}}, \\
& Q_{5}^{(5)}=\mathfrak{q} \sqrt{\frac{M_{1} M_{2} M_{3} M_{4} M_{7} M_{8} M_{9} M_{10}}{M_{5} M_{6}}}, \quad Q_{6}^{(5)}=\mathfrak{q}^{2} \frac{M_{5}}{M_{6}}, \quad Q_{7}^{(5)}=\mathfrak{q}^{2} M_{7} M_{8} M_{9}, \quad Q_{8}^{(5)}=\mathfrak{q}^{2} \frac{M_{10}}{M_{6}}, \\
& Q_{1}^{(6)}=A_{3} M_{8} M_{9}, \quad Q_{2}^{(6)}=\frac{M_{10}}{M_{7}}, \quad Q_{3}^{(6)}=\mathfrak{q} \sqrt{\frac{M_{1} M_{2} M_{3} M_{4} M_{5} M_{6} M_{8} M_{9}}{M_{7} M_{10}}}, \quad Q_{4}^{(6)}=\mathfrak{q} \sqrt{\frac{M_{5} M_{6} M_{8} M_{9} M_{10}}{M_{1} M_{2} M_{3} M_{4} M_{7}}}, \\
& Q_{5}^{(6)}=\mathfrak{q} \sqrt{\frac{M_{1} M_{2} M_{3} M_{4} M_{6} M_{8} M_{9} M_{10}}{M_{5} M_{7}}}, \quad Q_{6}^{(6)}=\mathfrak{q}^{2} \frac{M_{5}}{M_{7}}, \quad Q_{7}^{(6)}=\mathfrak{q}^{2} M_{6} M_{8} M_{9}, \quad Q_{8}^{(6)}=\mathfrak{q}^{2} \frac{M_{10}}{M_{7}}, \\
& Q_{1}^{(7)}=\frac{M_{9}}{A_{1}}, \quad Q_{2}^{(7)}=\frac{M_{10}}{M_{8}}, \quad Q_{3}^{(7)}=\mathfrak{q} \sqrt{\frac{M_{1} M_{2} M_{3} M_{4} M_{5} M_{6} M_{7} M_{9}}{M_{8} M_{10}}}, \quad Q_{4}^{(7)}=\mathfrak{q} \sqrt{\frac{M_{5} M_{6} M_{7} M_{9} M_{10}}{M_{1} M_{2} M_{3} M_{4} M_{8}}}, \\
& Q_{5}^{(7)}=\mathfrak{q} \sqrt{\frac{M_{1} M_{2} M_{3} M_{4} M_{6} M_{7} M_{9} M_{10}}{M_{5} M_{8}}}, \quad Q_{6}^{(7)}=\mathfrak{q}^{2} \frac{M_{5}}{M_{8}}, \quad Q_{7}^{(7)}=\mathfrak{q}^{2} M_{6} M_{7} M_{9}, \quad Q_{8}^{(7)}=\mathfrak{q}^{2} \frac{M_{10}}{M_{8}}, \\
& Q_{3}^{(8)}=\mathfrak{q} \sqrt{\frac{M_{1} M_{2} M_{3} M_{4} M_{5} M_{6} M_{7} M_{8}}{M_{9} M_{10}}}, \quad Q_{4}^{(8)}=\mathfrak{q} \sqrt{\frac{M_{5} M_{6} M_{7} M_{8} M_{10}}{M_{1} M_{2} M_{3} M_{4} M_{9}}}, \\
& Q_{5}^{(8)}=\mathfrak{q} \sqrt{\frac{M_{1} M_{2} M_{3} M_{4} M_{6} M_{7} M_{8} M_{10}}{M_{5} M_{9}}}, \quad Q_{6}^{(8)}=\mathfrak{q}^{2} \frac{M_{5}}{M_{9}}, \quad Q_{7}^{(8)}=\mathfrak{q}^{2} M_{6} M_{7} M_{8}, \quad Q_{8}^{(8)}=\mathfrak{q}^{2} \frac{M_{10}}{M_{9}} .
\end{aligned}
$$

Here $M_{i}(i=1, \ldots, 10)$ denote the position of horizontal lines measured from "SU(3)" origin (see figure 12). To convert e.g. from Sp origin to SU origin, we have to multiply $\mathfrak{q}^{1 / 2} \Lambda_{\mathrm{SU}(3)}$ for all variables $x_{I}$ and $M_{i}$. We note that the other Kähler parameters $Q_{j}^{(i)}$ with large $j$ is obtained by

$$
Q_{j+6}^{(i)}=\mathfrak{q}^{2} Q_{j}^{(i)} \quad(\text { for } i=1,2, \cdots, 8, \quad j \geq 3) .
$$



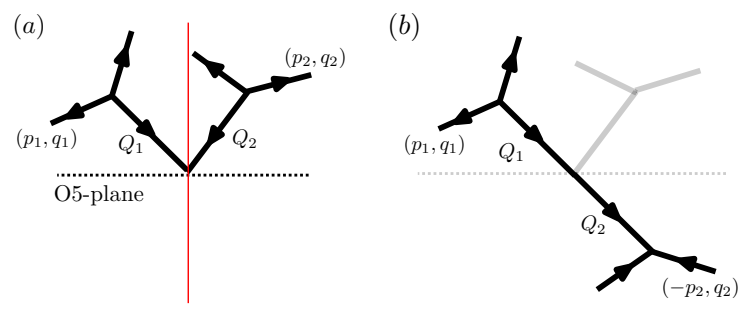

Figure 13. The rule how to interpret the web diagram with the O5-plane depicted in figure (a). With respect to the O5-plane, we flip the sign of the first elements of the charge vectors.

These are all the Kähler parameters which are necessary to compute the 2-instanton contribution.

\section{C $(p, q) 5$-brane web with O5-planes and topological vertex}

\section{C.1 Topological vertex formalism with O5-planes}

Here we summarize the computation rule of the partition function of the Sp gauge theories realized by the web diagrams with O5-planes discussed in [24]. We decompose the web diagram into the left side and right side about the red line. Then, we flip the sign of the first elements of the charge vectors, $(p, q) \rightarrow(-p, q)$, whose graphical meaning is to fold one side along the O5-plane. Here we fold the right side. The glueing rule of the vertices across the O5-plane is given as follows,

$$
\sum_{\mu} C_{\lambda_{1} \mu \nu_{1}} C_{\lambda_{2} \mu^{t} \nu_{2}}\left(+Q_{1} Q_{2}\right)^{|\mu|} f_{\mu}^{\mathfrak{n}}, \mathfrak{n}=\left(p_{2},-q_{2}\right) \wedge\left(p_{1}, q_{1}\right)+1
$$

Now we are ready to write down the partition function of the $\operatorname{Sp}(2)$ gauge theory with 10 flavors whose web diagram is given in figure 4 ,

$$
\begin{aligned}
& Z^{\mathrm{Sp}(2)+10 \mathrm{~F}}=\sum_{\left\{\nu_{i}\right\},\left\{\mu_{i}\right\}}\left(\frac{\mathfrak{q}^{\prime 2}}{x_{1}^{\prime 2}}\right)^{\left|\mu_{1}\right|}\left(\frac{\prod_{i=3}^{5} y_{i}^{\prime} y_{i+5}^{\prime}}{x_{2}^{\prime 2} x_{3}^{\prime 2}}\right)^{\left|\mu_{2}\right|}\left({x^{\prime}}_{3}^{2}\right)^{\left|\mu_{3}\right|} f_{\mu_{1}}^{-3} f_{\mu_{3}}^{3} \\
& \times\left(-x^{\prime}{ }_{1} y_{1}^{\prime-1}\right)^{\left|\nu_{1}\right|}\left(-y_{1}^{\prime} y_{2}^{\prime-1}\right)^{\left|\nu_{2}\right|}\left(-y_{2}^{\prime} x_{2}^{\prime-1}\right)^{\left|\nu_{3}\right|}\left(-x_{2}^{\prime} y_{3}^{\prime-1}\right)^{\left|\nu_{4}\right|}\left(-y_{3}^{\prime} y_{4}^{\prime-1}\right)^{\left|\nu_{5}\right|}\left(-y_{4}^{\prime} y_{5}^{\prime-1}\right)^{\left|\nu_{6}\right|} \\
& \times\left(-y_{5}^{\prime} x_{3}^{\prime-1}\right)^{\left|\nu_{7}\right|}\left(-x_{3}^{\prime 2}{ }^{2}\right)^{\left|\nu_{8}\right|}\left(-y_{6}^{\prime} x_{3}^{\prime-1}\right)^{\left|\nu_{9}\right|}\left(-y_{7}^{\prime} y_{6}^{\prime-1}\right)^{\left|\nu_{10}\right|}\left(-y_{8}^{\prime} y_{7}^{\prime-1}\right)^{\left|\nu_{11}\right|} \times\left(-x_{2}^{\prime} y_{8}^{\prime-1}\right)^{\left|\nu_{12}\right|} \\
& \times\left(-y_{9}^{\prime} x_{2}^{\prime-1}\right)^{\left|\nu_{13}\right|}\left(-y_{10}^{\prime} y_{9}^{\prime-1}\right)^{\left|\nu_{14}\right|}\left(-x^{\prime}{ }_{1} y^{\prime-1}\right)^{\left|\nu_{15}\right|}\left(-\mathfrak{q}^{\prime 2} x_{1}^{\prime-2}\right)^{\left|\nu_{16}\right|} \\
& \times f_{\nu_{2}}^{-1} f_{\nu_{5}}^{-1} f_{\nu_{6}}^{-1} f_{\nu_{10}}^{-1} f_{\nu_{11}}^{-1} f_{\nu_{14}}^{-1} \\
& \times C_{\nu_{1} \nu_{16}^{t} \mu_{1}} C_{\nu_{1}^{t} \nu_{2}^{t} \emptyset} C_{\nu_{2} \nu_{3}^{t} \emptyset} C_{\nu_{4}^{t} \nu_{3} \mu_{2}} C_{\nu_{4} \nu_{5}^{t} \emptyset} C_{\nu_{5} \nu_{6}^{\dagger} \emptyset} C_{\nu_{6} \nu_{7}^{t} \emptyset} C_{\nu_{8} \nu_{7} \mu_{3}} \\
& \times C_{\nu_{8}^{t} \nu_{9}^{t} \mu_{3}^{t}} C_{\nu_{10} \nu_{9} \emptyset} C_{\nu_{11} \nu_{10}^{t} \emptyset} C_{\nu_{12} \nu_{11}^{t} \emptyset} C_{\nu_{12}^{t} \nu_{13} \mu_{2}^{t}} C_{\nu_{14} \nu_{13}^{t} \emptyset} C_{\nu_{15}^{t} \nu_{14}^{t} \emptyset} C_{\nu_{15} \nu_{16} \mu_{1}^{t}} .
\end{aligned}
$$

The efficient way to calculate the partition function is to use the operator formalism explained in [43]. After some computations, one finds obtain (2.12). To reach the result, we use the analytic continuation formula,

$$
\Theta_{\mu \nu}(Q) \rightarrow Q^{|\mu|+|\nu|} f_{\mu} f_{\nu} \Theta_{\mu^{t} \nu^{t}}\left(Q^{-1}\right)
$$




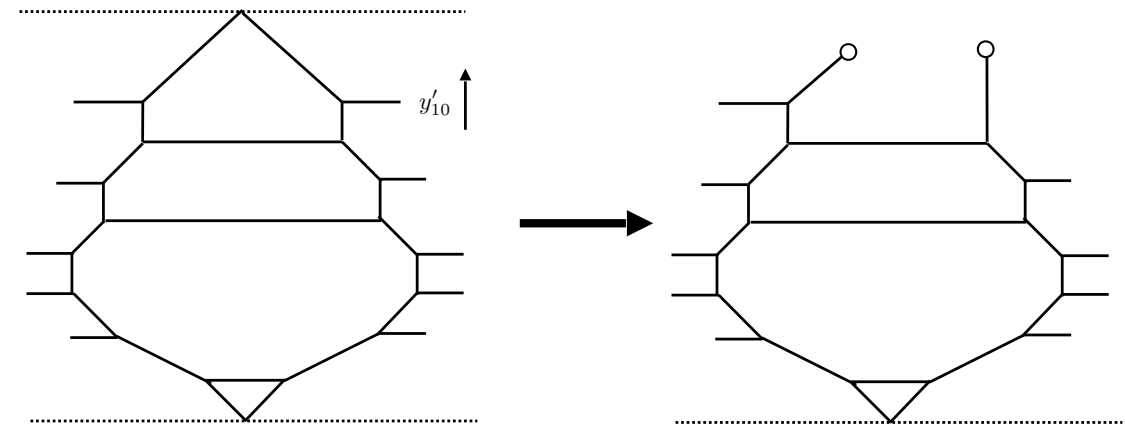

Figure 14. A pictorial explanation of the decoupling limit from 10 flavors to 9 flavors.

\section{C.2 The partition function of SU $(N+1)_{0}+(2 N+6) \mathrm{F}$}

In section 2.2 , we have calculated the partition function of $5 \mathrm{~d} \mathcal{N}=1 \mathrm{SU}(3)$ gauge theory with 10 flavors. Then, after the usual Higgsing, we have found the partition function of the $\mathrm{SU}(2)$ gauge theory with 8 flavors in section 3.1. Based on these results, we propose the partition function of $\mathrm{SU}(N+1)_{0}$ gauge theory with $2 N+6$ flavors without any computations,

$$
\begin{aligned}
Z^{\mathrm{SU}(N+1)+(2 N+6) \mathrm{F}}= & Z_{\text {mass }}^{2 N+6} \sum_{\mu_{1, \ldots, N+1}} \prod_{I=1}^{N+1}\left(\mathfrak{q} \Lambda_{\mathrm{SU}(N+1)}^{-2} A_{I}^{-3-N}\right)^{\left|\mu_{I}\right|} f_{\mu_{I}}^{-3-N} \\
& \times \prod_{I=1}^{N+1}\left(\prod_{i=1}^{2 N+6} \frac{\Theta_{\mu_{I} \varnothing}\left(A_{I} M_{i}^{-1}\right)}{\Theta_{\mu_{I} \varnothing}\left(\mathfrak{q} A_{I} M_{i} \Lambda_{\mathrm{SU}(N+1)}^{2}\right)} \prod_{J=1}^{N+1} \frac{\Theta_{\mu_{I} \mu_{J}}\left(\mathfrak{q} A_{I} A_{J} \Lambda_{\mathrm{SU}(N+1)}^{2}\right)}{\Theta_{\mu_{I} \mu_{J}^{t}}\left(A_{I} A_{J}^{-1}\right)}\right), \\
Z_{\mathrm{mass}}^{2 N+6}= & \prod_{i, j=1}^{\infty} \frac{1}{\left(q^{i+j-1} \mathfrak{q}: \mathfrak{q}\right)_{\infty}^{N+3}} \\
& \times \frac{\prod_{1 \leq i, j \leq N+3} \Theta_{\varnothing \varnothing}\left(\mathfrak{q} M_{i} M_{j+N+3} \Lambda_{\mathrm{SU}(N+1)}^{2}\right)}{\prod_{1 \leq i<j \leq N+3} \Theta_{\varnothing \varnothing}\left(M_{i} M_{j}^{-1}\right) \Theta_{\varnothing \varnothing}\left(M_{i+N+3} M_{j+N+3}^{-1}\right)},
\end{aligned}
$$

where $\Lambda_{\mathrm{SU}(N+1)}=\prod_{i=1}^{2 N+6} M_{i}^{-1 / 4}$ and the Coulomb branch moduli satisfy $\prod_{I=1}^{N+1} A_{I, \mathrm{SU}(N+1)}=1$.

\section{Flavor decoupling limit}

To consider what global symmetry is preserved in the presence of the defect in the $5 \mathrm{~d}$ gauge theories with $N_{f}<10$ flavors, we shall take the decoupling limit and consider the defect Higgsing. The gauge theory with 9 flavors can be obtained by the following decoupling limit,

$$
y_{10}^{\prime} \rightarrow 0, \quad \mathfrak{q}^{\prime} \rightarrow 0, \quad \frac{\mathfrak{q}^{\prime 2}}{y_{10}^{\prime}}=\mathfrak{q}_{9}^{\prime 2}(\text { fixed })
$$

where $\mathfrak{q}_{9}^{\prime}$ is the instanton factors of $5 \mathrm{~d} \operatorname{Sp}(2)$ gauge theory with 9 flavors. Diagrammatically, the decoupling limit is given in figure 14. The left side of this web diagram and the one in figure 4 are equivalent under the flop transition [44-46]. Then, the decoupling limit 
is done by removing one of the external legs from the web diagram, and the resulting web diagram is the right side of figure 14, where the white circles denote the D7-branes. Since we remove one of the O5-plane, this limit also implies the decompactification limit.

From the elliptic genus of $6 \mathrm{~d} \mathrm{Sp}(1)$ gauge theory with 10 flavors (3.39) with the duality map (3.31), we have the partition function of $5 \mathrm{~d} \mathrm{Sp}(2)$ gauge theory with 9 flavors,

$$
Z^{\prime \mathrm{Sp}(2)+9 \mathrm{~F}}=Z_{(0), 9 \mathrm{~F}}^{\prime}+Z_{(1), 9 \mathrm{~F}}^{\prime} A_{1}^{\prime}+\mathcal{O}\left({A^{\prime}}_{1}^{2}\right)
$$

with

$$
\begin{aligned}
& Z_{(0), 9 \mathrm{~F}}^{\prime}=\frac{1}{2} \frac{q}{(1-q)^{2}}\left(2\left({A^{\prime}}_{2}^{2}+{A^{\prime}}_{2}^{-2}\right)-\left({A^{\prime}}_{2}+{A^{\prime}}_{2}^{-1}\right)\left(\sum_{i=1}^{9}\left(y_{i}^{\prime}+y_{i}^{\prime-1}\right)+\left(\mathfrak{q}^{\prime}+\mathfrak{q}^{\prime-1}\right)\right)\right), \\
& Z_{(1), 9 \mathrm{~F}}^{\prime}=\frac{1}{(1-q)\left(1-q^{-1}\right)} \frac{\mathfrak{q}_{9}^{\prime 2}}{\left(1-{A^{\prime}}_{2}^{2}\right)\left(1-{A^{\prime}}_{2}^{-2}\right)}\left(Z_{(1), 9 \mathrm{~F}}^{\prime \mathrm{SOSU}}+Z_{(1), 9 \mathrm{~F}}^{\prime \mathrm{SU}}\right),
\end{aligned}
$$

where

$$
\begin{aligned}
Z_{(1), 9 \mathrm{~F}}^{\prime \mathrm{SO}, \mathrm{SU}} & =\left.\tilde{Z}_{(1)}^{\mathrm{SO}, \mathrm{SU}}\right|_{\tilde{y}_{1, \ldots, 9} \rightarrow y_{1, \ldots, 9}^{\prime}, \tilde{y}_{10} \rightarrow \mathfrak{q}_{9}^{\prime}, \tilde{A} \rightarrow A_{2}^{\prime},}, \\
Z_{(1), 9 \mathrm{~F}}^{\prime \mathrm{SU}} & =\left.\tilde{Z}_{(1)}^{\mathrm{SU}}\right|_{\tilde{y}_{1, \ldots, 9} \rightarrow y_{1, \ldots, 9}^{\prime}, \tilde{y}_{10} \rightarrow \mathfrak{q}_{9}^{\prime}, \tilde{A} \rightarrow A_{2}^{\prime}}
\end{aligned}
$$

Note that the elliptic genus for 1-string (3.39) is given as a series of $\mathfrak{q}^{\prime}$, however, (D.3) is exact results in $\mathfrak{q}^{\prime}$.

Based on (2.14), we find the duality map between $\mathrm{Sp}(2)$ and $\mathrm{SU}(3)$ gauge theories with 9 flavors is

$$
\begin{aligned}
& y_{i}^{\prime}=\mathfrak{q}^{1 / 2}\left(\prod_{j=1}^{9} M_{j}^{-1 / 4}\right) M_{i}(i=1, \ldots, 9), \\
& A_{i}^{\prime}=\mathfrak{q}^{1 / 2}\left(\prod_{j=1}^{9} M_{j}^{-1 / 4}\right) A_{i}(i=1,2), \quad \mathfrak{q}^{\prime}=\mathfrak{q}^{3 / 4}\left(\prod_{j=1}^{9} M_{j}^{1 / 8}\right) .
\end{aligned}
$$

We check the agreement of the partition functions between the $\mathrm{Sp}(2)$ and $\mathrm{SU}(3)$ gauge theories with 9 flavors under the duality map up to second order of $A_{1}$ and $\mathfrak{q}$. We also check that, by expanding the partition functions of $\operatorname{Sp}(2)$ and $\mathrm{SU}(3)$ gauge theory as a series of $A_{1}$ and $\mathfrak{q}$, and using the analytic continuation formula for (D.3), these partition functions have only positive power of $A_{1}$ and $\mathfrak{q}$.

By the defect Higgsing given by,

$$
A_{2}^{\prime}=q^{M} y_{3}^{\prime}=y_{8}^{\prime}
$$

which is derived from the Higgsing in $6 \mathrm{~d} \operatorname{Sp}(1)$ theory with 10 flavors (3.36) and the duality map (3.31), we obtain the $\mathrm{Sp}(1)$ gauge theory with 7 flavors in the presence of the defects, and $Z_{(1), 9 F}^{\prime S O}$ is given by

$$
\begin{aligned}
& Z_{(1), 9 \mathrm{~F}}^{\prime \mathrm{SO}, \mathrm{SU}} \rightarrow\left(-\left(q^{M} z^{\prime}-q^{-M} z^{\prime-1}\right)\left(q^{M / 2} z^{\prime}-q^{-M / 2} z^{\prime-1}\right) \chi_{\mathbf{1 2 8}}^{\mathrm{SO}(16)}\right. \\
& -\left(q^{M} z^{\prime}-q^{-M} z^{\prime-1}\right)\left(q^{M / 2}-q^{-M / 2}\right) \chi^{\prime \mathbf{S O}(16)}-\left(q^{M} z^{\prime}-q^{-M} z^{\prime-1}\right)^{2} \chi_{\mathbf{1 2 0}}^{\prime \mathrm{SO}(16)} \\
& \left.+\left(q^{M} z^{\prime}-q^{-M} z^{-1}\right)^{2}\left(q^{M / 2} z^{\prime}-q^{-M / 2} z^{\prime-1}\right)\left(q^{M / 2}-q^{-M / 2}\right) \chi_{\mathbf{1 6}}^{\prime \mathrm{SO}(16)}\right),
\end{aligned}
$$




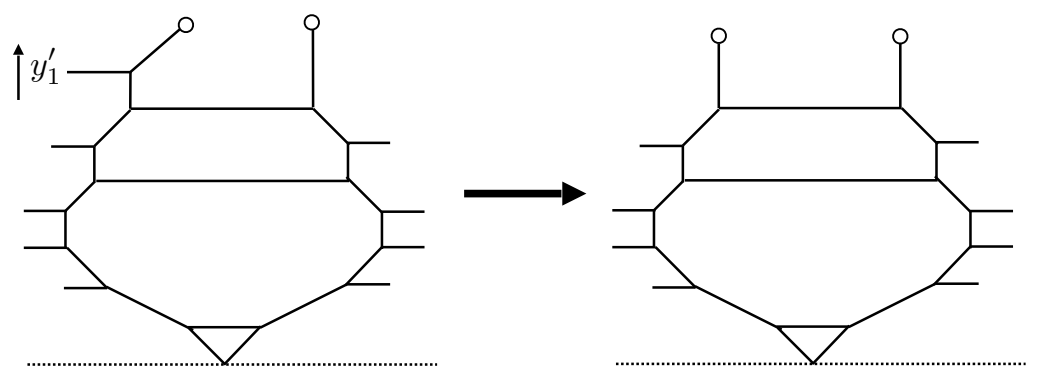

Figure 15. A decoupling limit from 9 flavors to 8 flavors.

where $z^{\prime}=y_{3}^{\prime}$ is the brane moduli of $5 \mathrm{~d} \mathrm{Sp}(1)$ gauge theory. Here, prime of $\chi_{\mathbf{1 2 8}, \mathbf{1 2 8}, \mathbf{1 2 0 , 1 6}}^{\mathrm{SO}(16)}$ denote the characters of $\mathrm{SO}(16)$ which are defined by replacing $6 \mathrm{~d} \mathrm{Sp}(1)$ gauge theory fugacities $\left\{\tilde{y}_{i}\right\}_{i \in \mathcal{I}}$ of (3.44) with the $5 \mathrm{~d} \mathrm{Sp}(2)$ gauge theory fugacities and instanton factor $\left\{y_{i}^{\prime}\right\}_{i \in \mathcal{I}_{9 \mathrm{~F}}}, \mathfrak{q}_{9}^{\prime}$ where $\mathcal{I}_{9 \mathrm{~F}}=\{1,2,4,5,6,7,9\}$. When we set $M=0, \mathcal{F}_{(1)}^{\prime}$ is given by $E_{8}$ character, so that the global symmetry is $E_{8}$ symmetry which is the enhanced symmetry of SU(2) gauge theory with 7 flavors discussed in [47]. Therefore, we conclude that the enhanced symmetry $E_{8}$ is broken to $\mathrm{SO}(16)$ by the defects.

We further consider one more flavor decoupling as in figure 15 whose limit is given by

$$
y_{1}^{\prime} \rightarrow 0, \quad \mathfrak{q}_{9}^{\prime} \rightarrow 0, \quad \frac{\mathfrak{q}_{9}^{\prime 2}}{y_{1}^{\prime}}=\mathfrak{q}_{8}^{\prime 2}(\text { fixed })
$$

In the same way as above case, we denote $\mathfrak{q}_{8}^{\prime}$ as the instanton factor of $5 \mathrm{~d} \operatorname{Sp}(2)$ gauge theory with 8 flavors. The resulting partition function of $5 \mathrm{~d} \operatorname{Sp}(2)$ with 8 flavors is,

$$
Z^{\prime \mathrm{Sp}(2)+8 \mathrm{~F}}=Z_{(0), 8 \mathrm{~F}}^{\prime}+Z_{(1), 8 \mathrm{~F}}^{\prime} A_{1}^{\prime}+\mathcal{O}\left(A_{1}^{\prime 2}\right),
$$

with

$$
\begin{aligned}
& Z_{(0), 8 \mathrm{~F}}^{\prime}=\frac{1}{2} \frac{q}{(1-q)^{2}}\left(2\left({A^{\prime}}_{2}^{2}+{A^{\prime}}_{2}^{-2}\right)-\left(A_{2}^{\prime}+A_{2}^{\prime-1}\right) \sum_{i=2}^{9}\left(y_{i}^{\prime}+y_{i}^{\prime-1}\right)\right), \\
& Z_{(1), 8 \mathrm{~F}}^{\prime}=\frac{1}{(1-q)\left(1-q^{-1}\right)} \frac{\mathfrak{q}_{8}^{\prime}}{\left(1-{A^{\prime}}_{2}^{2}\right)\left(1-{A^{\prime}}_{2}^{-2}\right)}\left(Z_{(1), 8 \mathrm{~F}}^{\prime A}+Z_{(1), 8 \mathrm{~F}}^{\prime B}\right),
\end{aligned}
$$

and

$$
\begin{aligned}
Z_{(1), 8 \mathrm{~F}}^{\prime \mathrm{SOSU}}= & -\chi^{\prime \mathrm{SO}(16)} \chi_{1 / 2}^{\mathrm{SU}(2)}\left(A_{2}^{\prime}\right)+2 \chi_{\mathbf{1 2 8}}^{\mathrm{SO}(16)} \\
& -\chi_{\mathbf{1 6}}^{\prime \mathrm{SO}(16)} \chi_{1 / 2}^{\mathrm{SU}(2)}\left(\mathfrak{q}_{8}^{\prime}\right) \chi_{1}^{\mathrm{SU}(2)}\left(A_{2}^{\prime}\right)+3 \chi_{\mathbf{1 6}}^{\prime \mathrm{SO}(16)} \chi_{1 / 2}^{\mathrm{SU}(2)}\left(\mathfrak{q}_{8}^{\prime}\right), \\
Z_{(1), 8 \mathrm{~F}}^{\prime \mathrm{SU}}= & 2 \chi_{1 / 2}^{\mathrm{SU}(2)}\left(\mathfrak{q}_{8}^{\prime}\right) \chi_{3 / 2}^{\mathrm{SU}(2)}\left(A_{2}^{\prime}\right)-4 \chi_{1 / 2}^{\mathrm{SU}(2)}\left(\mathfrak{q}_{8}^{\prime}\right) \chi_{1 / 2}^{\mathrm{SU}(2)}\left(A_{2}^{\prime}\right) .
\end{aligned}
$$

Then, by defining the invariant Coulomb moduli $A_{1, \text { inv }}^{\prime}$ as

$$
A_{1, \mathrm{inv}}^{\prime}=\mathfrak{q}_{8}^{\prime} A_{1}^{\prime},
$$

the partition function has $\mathrm{SO}(16) \times \mathrm{SU}(2)$ invariance. 

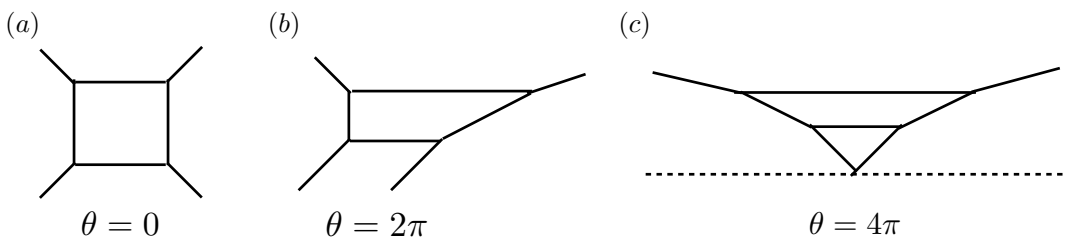

Figure 16. The web diagram descriptions of pure $\mathrm{SU}(2)$ gauge theories with $\theta=0,2 \pi$, and $4 \pi$.

By the defect Higgsing (D.6), we have

$$
\begin{aligned}
Z_{(1), 8 \mathrm{~F}}^{\prime \mathrm{SOSU}} \rightarrow & -\left(q^{M} z^{\prime}-q^{-M} z^{{ }^{-1}}\right)\left(q^{M / 2} z^{\prime}-q^{-M / 2} z^{\prime-1}\right) \chi_{\overline{\mathbf{3 2}}}^{\mathrm{SO}(12)} \\
& -\left(q^{M} z^{\prime}-q^{-M} z^{\prime-1}\right)\left(q^{M / 2}-q^{-M / 2}\right) \chi_{\mathbf{3 2}}^{\mathrm{SO}(12)} \\
& -\left(q^{M} z^{\prime}-q^{-M} z^{\prime-1}\right)^{2} \chi_{\mathbf{1 2}}^{\mathrm{SO}(12)} \chi_{1 / 2}^{\mathrm{SU}(2)}\left(\mathfrak{q}^{\prime}{ }_{8}\right),
\end{aligned}
$$

where we write the contribution only involving $\mathrm{SO}(12)$ characters defined by

$$
\begin{aligned}
\chi_{\mathbf{3 2}}^{\mathrm{SO}(12)} & =\frac{1}{2}\left(\prod_{i \in \mathcal{I}^{\prime}}\left(y_{i}^{\prime 1 / 2}+y_{i}^{\prime-1 / 2}\right)+\prod_{i \in \mathcal{I}^{\prime}}\left(y_{i}^{\prime 1 / 2}-y_{i}^{\prime-1 / 2}\right)\right), \\
\chi_{\overline{\mathbf{3 O}}^{\mathrm{SO}}(12)} & =\frac{1}{2}\left(\prod_{i \in \mathcal{I}^{\prime}}\left(y_{i}^{\prime 1 / 2}+y_{i}^{\prime-1 / 2}\right)-\prod_{i \in \mathcal{I}^{\prime}}\left(y_{i}^{\prime 1 / 2}-y_{i}^{\prime-1 / 2}\right)\right), \\
\chi_{\mathbf{1 2}}^{\mathrm{SO}(12)} & =\sum_{i \in \mathcal{I}^{\prime}}\left(y_{i}^{\prime}{ }_{i}+y_{i}^{\prime-1}\right) . \quad\left(\mathcal{I}^{\prime}=\{2,4,5,6,7,9\}\right)
\end{aligned}
$$

When we set $M=0$, (D.13) can be expressed as $E_{7}$ characters,

$$
(\mathrm{D} .13)=-\left(z^{\prime}-z^{\prime-1}\right)^{2}\left(\chi_{\overline{\mathbf{3 2}}}^{\mathrm{SO}(12)}+\chi_{1 / 2}^{\mathrm{SU}(2)}\left(\mathfrak{q}_{8}^{\prime}\right) \chi_{\mathbf{1 2}}^{\mathrm{SO}(12)}\right)=-\left(z^{\prime}-z^{\prime-1}\right)^{2} \chi_{\mathbf{5 6}}^{E_{7}}
$$

\section{E Defects on pure SU(2) theories with different discrete theta angles}

In this appendix, we explain how the defect affects the symmetry. As an example, we shall consider $5 \mathrm{~d} \mathcal{N}=1$ pure $\mathrm{SU}(2)$ gauge theories with theta angle $\theta=0, \theta=2 \pi$, and $\theta=4 \pi$ whose web diagram descriptions are given in figure 16. Without the defect, these theories are equivalent through the Hanany-Witten transition. A way to see the equivalence is to compare the partition functions of these theories. After some computation, one can show

$$
Z_{\theta=0}=\frac{Z_{\theta=2 \pi}}{Z_{E}}=Z_{\theta=4 \pi},
$$

where $Z_{\theta=0,2 \pi, 4 \pi}$ denote the partition functions of pure $\mathrm{SU}(2)$ gauge theories with $\theta=$ $0,2 \pi, 4 \pi$, and $Z_{E}$ denotes the extra factor. In this case, $Z_{E}$ corresponds to the strings attached between parallel external lines in figure 16 (b).

In the presence of the defect, however, the situation is changed: we need to include the contribution coming from the framing. To see explicitly, let us consider following web 
(a)

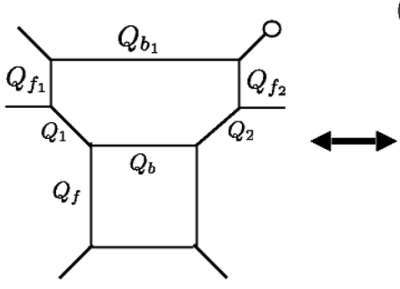

$(b)$

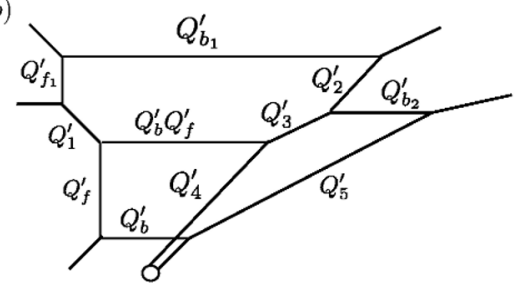

Figure 17. The web diagrams which are dual each other.

diagrams as an example of the duality between $\theta=0$ and $2 \pi$. The web diagrams (a) and (b) reduce to $\mathrm{SU}(2)$ gauge theory with $\theta=0$ and $\theta=2 \pi$ in the presence of the defects after the geometric transition. For simplicity, here we consider the insertion of single brane which is realized by setting the Kähler moduli as follows,

$$
Q_{f_{1}}=q^{-1}, Q_{f_{2}}=1, Q_{f_{1}}^{\prime}=q^{-1}, Q_{3}^{\prime}=1 .
$$

Then, we find

$$
\begin{aligned}
& Z^{(a)}=\sum_{\mu_{b_{1}, 2}}\left(-Q_{b}\right)^{\left|\mu_{b_{1}}\right|+\left|\mu_{b_{2}}\right|} f_{\mu_{b_{1}}} f_{\mu_{b_{2}}}^{-1} s_{\mu_{b_{1}}}\left(q^{-\rho}\right) s_{\mu_{b_{1}}^{t}}\left(q^{-\rho}\right) s_{\mu_{b_{2}}}\left(q^{-\rho}\right) s_{\mu_{b_{2}}^{t}}\left(q^{-\rho}\right) \\
& \times \prod_{i, j=1}^{\infty} \frac{1}{\left(1-Q_{f} q^{i+j-\mu_{b_{1}, i}-\mu_{b_{2}, j}^{t}-1}\right)^{2}} \times \prod_{i, j=1}^{\infty} \frac{\left(1-Q_{1} q^{i+j-\mu_{b_{1, j}}^{t}-1}\right)\left(1-Q_{1} Q_{f} q^{i+j-\mu_{b_{2}, j}^{t}-1}\right)}{\left(1-Q_{1} q^{i+j-\mu_{b_{1}, j}^{t}-2}\right)\left(1-Q_{1} Q_{f} q^{i+j-\mu_{b_{2}, j}^{t}-2}\right)} \\
& Z^{(b)}=\sum_{\mu_{b_{1}, 2}, \mu_{b_{1}}^{\prime}}\left(-Q_{b}^{\prime} Q_{f}^{\prime 2}\right)^{\left|\mu_{b_{1}}\right|}\left(-Q_{b}^{\prime}\right)^{\left|\mu_{b_{2}}\right|} f_{\mu_{b_{1}}}^{3} f_{\mu_{b_{2}}} s_{\mu_{b_{1}}}\left(q^{-\rho}\right) s_{\mu_{b_{1}}^{t}}\left(q^{-\rho}\right) s_{\mu_{b_{2}}}\left(q^{-\rho}\right) s_{\mu_{b_{2}}^{t}}\left(q^{-\rho}\right) \\
& \times \prod_{i, j=1}^{\infty} \frac{1}{\left(1-Q_{f}^{\prime} q^{i+j-\mu_{b_{1}, i}-\mu_{b_{2}, j}^{t}-1}\right)^{2}} \\
& \times\left(-Q_{b_{1}}^{\prime}\right)^{\mid \mu_{b_{1}}^{\prime}} \mid f_{\mu_{b_{1}}^{\prime}}^{3} s_{\mu_{b_{1}}^{\prime}}\left(q^{-\rho}\right) \prod_{i, j=1}^{\infty} \frac{\left(1-Q_{1} q^{i+j-\mu_{b_{1}, j}^{t}-1}\right)\left(1-Q_{1} Q_{f} q^{i+j-\mu_{b_{2}, j}^{t}-1}\right)}{\left(1-Q_{1} q^{i+j-\mu_{b_{1}, i}^{\prime}-\mu_{b_{1}, j}^{t}-2}\right)\left(1-Q_{1} Q_{f} q^{i+j-\mu_{b_{1}, i}^{\prime}-\mu_{b_{2}, j}^{t}-2}\right)},
\end{aligned}
$$

where we omit the contributions which is independent of the Kähler moduli, and the summation of $\mu_{b_{1}}^{\prime}$ in (E.3b) takes only the Young diagrams that have single row, $\mu_{b_{1}}^{\prime}=$ $\{n\}, n \in \mathbb{Z}_{\geq 0} \cdot{ }^{7}$ Then, we check the agreement of the partition functions,

$$
Z^{(a)}=\frac{Z^{(b)}}{Z_{\mathrm{E},(b)}}, Z_{\mathrm{E},(b)}=\prod_{i, j=1}^{\infty} \frac{1}{\left(1-Q_{b}^{\prime} q^{i+j-1}\right)},
$$

under the following relations,

$$
Q_{b} Q_{f}=Q_{b}^{\prime}, Q_{f}=Q_{f}^{\prime}, Q_{1}=Q_{1}^{\prime}, Q_{b_{1}}^{\prime}=q^{-1} Q_{b}^{\prime} Q_{f}^{\prime} Q_{1}^{\prime 2},
$$

as expected from Hanany-Witten transition. Diagrammatically, the relation is expressed as in figure 18. The factor $\left(-Q_{b_{1}}^{\prime}\right)^{\left|\mu_{b_{1}}^{\prime}\right|} f_{\mu_{b_{1}}^{\prime}}^{3} s_{\mu_{b_{1}}^{\prime}}\left(q^{-\rho}\right)$ comes from the trivalent diagram with the defect.

The interpretation is as follows. The brane configuration of D5-NS5-D7-brane system are given in table 1. Since the D7-branes have branch cut and affect the charges of D5-

\footnotetext{
${ }^{7}$ In the following, the summation of the Young diagram with prime takes only single row.
} 
(a)

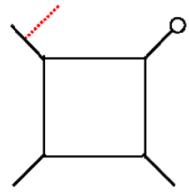

(b)

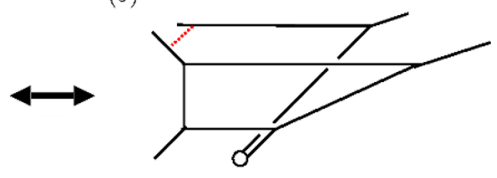

Figure 18. The web diagrams after the geometric transition.

\begin{tabular}{|c|c|c|c|c|c|c|c|c|c|c|}
\hline IIB & $X_{0}$ & $X_{1}$ & $X_{2}$ & $X_{3}$ & $X_{4}$ & $X_{5}$ & $X_{6}$ & $X_{7}$ & $X_{8}$ & $X_{9}$ \\
\hline D5 & $\circ$ & $\circ$ & $\circ$ & $\circ$ & $\circ$ & - & - & & & \\
\hline NS5 & $\circ$ & $\circ$ & $\circ$ & $\circ$ & $\circ$ & - & - & & & \\
\hline D7 & $\circ$ & $\circ$ & $\circ$ & $\circ$ & $\circ$ & & & $\circ$ & $\circ$ & $\circ$ \\
\hline
\end{tabular}

Table 1. The brane configuration of $(p, q)$-5-brane with D7-branes. The $(p, q)$-5-brane web is defined on the $X_{5,6}$-plane denoted by bar.

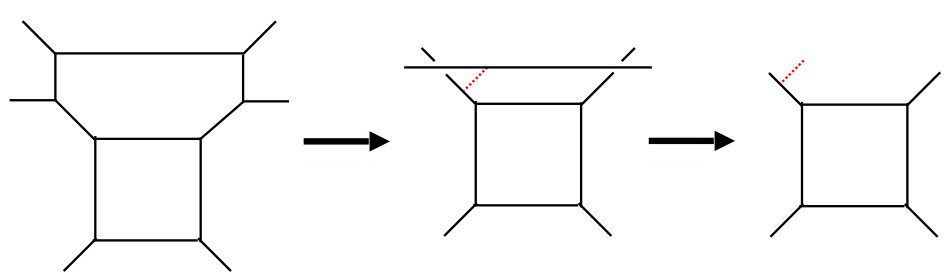

Figure 19. The process of geometric transition. We can remove the framing after setting some of Kähler moduli.

and NS5-branes, the shape of web diagrams change by moving the D7-branes. However, since the D7-branes do not affect the physical system, the partition function is invariant up to the extra factor.

In the topological strings, the defect corresponds to the topological brane wrapping on $S^{3}$ denoted by the red dashed line. Since the partition function of A-model topological string does not depend on the complex structure moduli, we can remove the framing by taking the size of $S^{3}$ to infinity as one can see in figure 19 .

However, when we put the D7-branes on the web diagram, the situation changes: since the D7-brane is extended infinitely except for $X_{5,6}$-plane, no matter how we separate off the framing from the remaining web diagram, the framing is affected by the movement of the D7-brane, and the framing acquires the non-trivial structure which provides the non-trivial contributions. This is why we have to consider the additional contribution coming from the framing to see the duality (see figure 20).

As another example, let us consider the duality between $\theta=0$ and $\theta=2 \pi$. Again, to find the correct duality, we utilize the geometric transition for the web diagram given in figure 21. Then, by setting some of the Kähler moduli as

$$
Q_{1}=q^{-1}, Q_{3}=1, Q_{b_{1}}^{\prime}=q^{-1}, Q_{b_{3}}^{\prime}=1,
$$

we find the partition functions of the theories in the presence of single defect, 


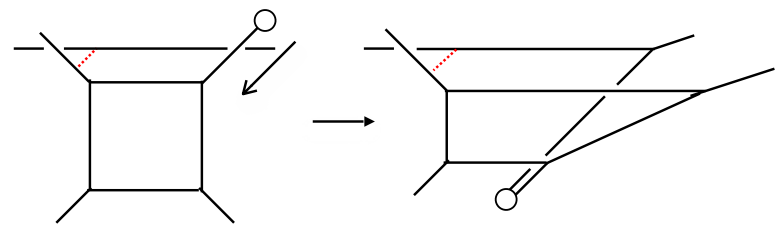

Figure 20. The Hanany-Witten transition in the presence of the defect. Since the D7-brane is extended along a direction, the D7-brane affects the framing.

(a)

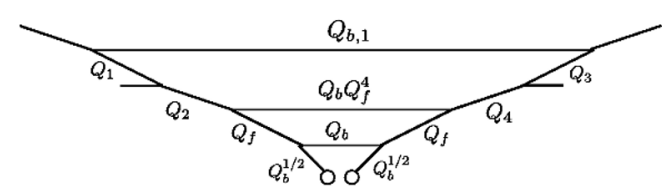

(b)

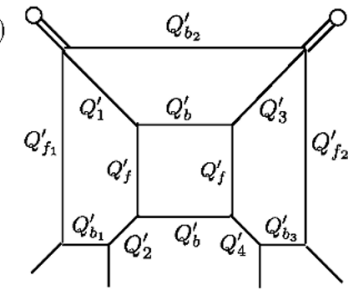

Figure 21. The web diagrams which reduce to $\mathrm{SU}(2)$ gauge theory with $\theta=4 \pi$ and $\theta=0$ in the presence of the defects after the geometric transition.

$$
\begin{aligned}
& Z^{(a)}=\sum_{\mu_{b_{1}, 2}}\left(-Q_{b} Q_{f}^{4}\right)^{\left|\mu_{b_{1}}\right|}\left(-Q_{b}\right)^{\left|\mu_{b_{2}}\right|} f_{\mu_{b_{1}}}^{-6} f_{\mu_{b_{2}}}^{4} q^{{ }^{\kappa} t_{b_{1}}^{t}+\mu_{b_{2}}} s_{\mu_{b_{1}}}^{2}\left(q^{-\rho}\right) s_{\mu_{b_{2}}^{t}}^{2}\left(q^{-\rho}\right) \\
& \times \prod_{i, j=1}^{\infty} \frac{\left(1-Q_{b} q^{i+j-\mu_{b_{2}, i}-\mu_{b_{2}, j}-1}\right)\left(1-Q_{b} Q_{f} q^{i+j-\mu_{b_{2}, i}-\mu_{b_{1}, j}^{t}-1}\right)^{2}\left(1-Q_{b} Q_{f}^{2} q^{i+j-\mu_{b_{1}, i}^{t}-\mu_{b_{1}, j}^{t}-1}\right)}{\left(1-Q_{f} q^{i+j-\mu_{b_{1}, i}-\mu_{b_{2}, j}^{t}-1}\right)^{2}} \\
& \times \prod_{i, j=1}^{\infty} \frac{\left(1-Q_{2} q^{i+j-\mu_{b_{1}, i}-1}\right)\left(1-Q_{2} Q_{f} q^{i+j-\mu_{b_{2}, j}^{t}}\right)\left(1-Q_{2} Q_{f} Q_{b} q^{i+j-\mu_{b_{2}, i}-1}\right)\left(1-Q_{2} Q_{f}^{2} Q_{b} q^{i+j-\mu_{b_{1}, i}-2}\right)\left(1-Q_{2} Q_{f} q^{i+j-\mu_{b_{2}, j}^{t}}{ }^{t}\right)\left(1-Q_{2} Q_{f} Q_{b} q^{i+j-\mu_{b_{2}, i}-2}\right)\left(1-Q_{2} Q_{f}^{2} Q_{b} q^{i+j-\mu_{b_{1}, j}^{t}}\right)}{\left({ }^{i}\right)}, \\
& Z^{(b)}=\sum_{\mu_{f_{1}, 2}, \mu_{f_{1}}^{\prime}}\left(-Q_{f}^{\prime}\right)^{\left|\mu_{f_{1}}\right|+\left|\mu_{f_{2}}\right|} f_{\mu_{f_{1}}} f_{\mu_{f_{2}}}^{-1} s_{\mu_{f_{1}}}\left(q^{-\rho}\right) s_{\mu_{f_{1}}^{t}}\left(q^{-\rho}\right) s_{\mu_{f_{2}}}\left(q^{-\rho}\right) s_{\mu_{f_{2}}^{t}}\left(q^{-\rho}\right) \\
& \times \prod_{i, j=1}^{\infty} \frac{1}{\left(1-Q_{b}^{\prime} q^{i+j-\mu_{f_{1}, i}-\mu_{f_{2}, j}^{t}-1}\right)^{2}} \\
& \times\left(-Q_{f_{1}}^{\prime}\right)^{\left|\mu_{f_{1}}^{\prime}\right|} f_{\mu_{f_{1}}^{\prime}}^{2} s_{\mu_{f_{1}}^{\prime}}\left(q^{-\rho}\right) \\
& \times \prod_{i, j=1}^{\infty} \frac{\left(1-Q_{2}^{\prime} q^{i+j-\mu_{f_{1}, j}^{t}-1}\right)\left(1-Q_{2}^{\prime} Q_{b}^{\prime} q^{i+j-\mu_{f_{2}, j}^{t}-1}\right)}{\left(1-Q_{2}^{\prime} q^{i+j-\mu_{f_{1}, i}^{\prime}-\mu_{f_{1}, j}^{t}-2}\right)\left(1-Q_{2}^{\prime} Q_{b}^{\prime} q^{i+j-\mu_{f_{1}, i}^{\prime}-\mu_{f_{2}, j}^{t}-2}\right)\left(1-Q_{b_{2}}^{\prime} q^{i+j-\mu_{f_{1}, i}^{\prime}-1}\right)} .
\end{aligned}
$$

Then we find

$$
Z^{(a)}=\frac{Z^{(b)}}{Z_{\mathrm{E},(b)}}, Z_{\mathrm{E},(b)}=\prod_{i, j=1}^{\infty} \frac{1}{\left(1-Q_{2}^{\prime 2} Q_{b}^{\prime} q^{i+j-2}\right)},
$$

under following relation,

$$
\frac{Q_{b}}{Q_{f}^{2}}=Q_{b}^{\prime}, Q_{f}=Q_{f}^{\prime}, Q_{2}=Q_{2}^{\prime}, Q_{f_{1}}^{\prime}=q^{-1} Q_{2}^{\prime 2} Q_{f}^{\prime}, Q_{b_{2}}^{\prime}=q^{-2} Q_{2}^{\prime 2} Q_{f}^{\prime} .
$$

The corresponding web diagrams are as follows. 
$(a)$

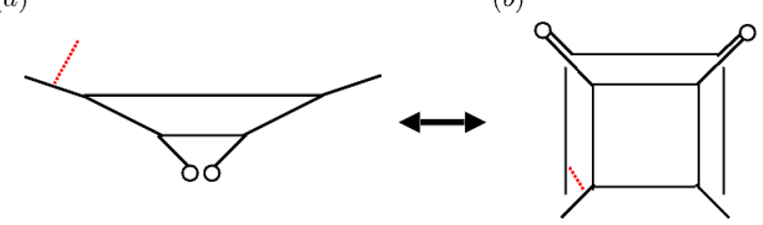

Figure 22. The web diagrams after the geometric transition. Since we move two D7-branes, the shape of framing after moving the D7-branes is more complicated than previous case depicted in figure 20 .

Open Access. This article is distributed under the terms of the Creative Commons Attribution License (CC-BY 4.0), which permits any use, distribution and reproduction in any medium, provided the original author(s) and source are credited.

\section{References}

[1] S. Gukov and E. Witten, Gauge theory, ramification, and the geometric Langlands program, hep-th/0612073 [INSPIRE].

[2] S. Gukov and E. Witten, Rigid surface operators, Adv. Theor. Math. Phys. 14 (2010) 87 [arXiv:0804.1561] [INSPIRE].

[3] J. Gomis and S. Matsuura, Bubbling surface operators and S-duality, JHEP 06 (2007) 025 [arXiv:0704.1657] [INSPIRE].

[4] L.F. Alday, D. Gaiotto, S. Gukov, Y. Tachikawa and H. Verlinde, Loop and surface operators in $N=2$ gauge theory and Liouville modular geometry, JHEP 01 (2010) 113 [arXiv:0909.0945] [INSPIRE].

[5] L.F. Alday and Y. Tachikawa, Affine SL(2) conformal blocks from 4d gauge theories, Lett. Math. Phys. 94 (2010) 87 [arXiv:1005.4469] [InSPIRE].

[6] D. Gaiotto, L. Rastelli and S.S. Razamat, Bootstrapping the superconformal index with surface defects, JHEP 01 (2013) 022 [arXiv: 1207.3577] [INSPIRE].

[7] D. Gaiotto, S. Gukov and N. Seiberg, Surface defects and resolvents, JHEP 09 (2013) 070 [arXiv: 1307.2578] [INSPIRE].

[8] D. Gaiotto and H.-C. Kim, Surface defects and instanton partition functions, JHEP 10 (2016) 012 [arXiv: 1412.2781] [INSPIRE].

[9] B. Nazzal and S.S. Razamat, Surface defects in E-string compactifications and the van Diejen model, SIGMA 14 (2018) 036 [arXiv:1801.00960] [InSPIRE].

[10] S.H. Katz, A. Klemm and C. Vafa, Geometric engineering of quantum field theories, Nucl. Phys. B 497 (1997) 173 [hep-th/9609239] [INSPIRE].

[11] S. Katz, P. Mayr and C. Vafa, Mirror symmetry and exact solution of $4 D N=2$ gauge theories: 1, Adv. Theor. Math. Phys. 1 (1998) 53 [hep-th/9706110] [INSPIRE].

[12] R. Dijkgraaf and C. Vafa, Matrix models, topological strings, and supersymmetric gauge theories, Nucl. Phys. B 644 (2002) 3 [hep-th/0206255] [INSPIRE]. 
[13] T.J. Hollowood, A. Iqbal and C. Vafa, Matrix models, geometric engineering and elliptic genera, JHEP 03 (2008) 069 [hep-th/0310272] [INSPIRE].

[14] N.C. Leung and C. Vafa, Branes and toric geometry, Adv. Theor. Math. Phys. 2 (1998) 91 [hep-th/9711013] [INSPIRE].

[15] T. Dimofte, S. Gukov and L. Hollands, Vortex counting and Lagrangian 3-manifolds, Lett. Math. Phys. 98 (2011) 225 [arXiv:1006.0977] [inSPIRE].

[16] H. Awata, H. Fuji, H. Kanno, M. Manabe and Y. Yamada, Localization with a surface operator, irregular conformal blocks and open topological string, Adv. Theor. Math. Phys. 16 (2012) 725 [arXiv: 1008.0574] [INSPIRE].

[17] R. Gopakumar and C. Vafa, On the gauge theory/geometry correspondence, Adv. Theor. Math. Phys. 3 (1999) 1415 [hep-th/9811131] [INSPIRE].

[18] H. Ooguri and C. Vafa, Knot invariants and topological strings, Nucl. Phys. B 577 (2000) 419 [hep-th/9912123] [INSPIRE].

[19] H. Mori and Y. Sugimoto, Surface operators from M-strings, Phys. Rev. D 95 (2017) 026001 [arXiv: 1608.02849] [INSPIRE].

[20] B. Haghighat, A. Iqbal, C. Kozçaz, G. Lockhart and C. Vafa, M-strings, Commun. Math. Phys. 334 (2015) 779 [arXiv: 1305.6322] [INSPIRE].

[21] Y. Sugimoto, The enhancement of supersymmetry in M-strings, Int. J. Mod. Phys. A 31 (2016) 1650088 [arXiv: 1508.02125] [inSPIRE].

[22] S.-S. Kim, M. Taki and F. Yagi, Tao probing the end of the world, PTEP 2015 (2015) 083B02 [arXiv: 1504.03672] [INSPIRE].

[23] M. Aganagic, A. Klemm, M. Mariño and C. Vafa, The topological vertex, Commun. Math. Phys. 254 (2005) 425 [hep-th/0305132] [INSPIRE].

[24] S.-S. Kim and F. Yagi, Topological vertex formalism with O5-plane, Phys. Rev. D 97 (2018) 026011 [arXiv: 1709.01928] [INSPIRE].

[25] D. Gaiotto and H.-C. Kim, Duality walls and defects in $5 d N=1$ theories, JHEP 01 (2017) 019 [arXiv: 1506.03871] [INSPIRE].

[26] H. Hayashi, S.-S. Kim, K. Lee, M. Taki and F. Yagi, A new $5 d$ description of $6 d$ D-type minimal conformal matter, JHEP 08 (2015) 097 [arXiv: 1505.04439] [INSPIRE].

[27] Y. Yun, Testing 5d-6d dualities with fractional D-branes, JHEP 12 (2016) 016 [arXiv: 1607.07615] [INSPIRE].

[28] J. Kim, S. Kim, K. Lee, J. Park and C. Vafa, Elliptic genus of E-strings, JHEP 09 (2017) 098 [arXiv: 1411.2324] [INSPIRE].

[29] H. Hayashi, S.-S. Kim, K. Lee and F. Yagi, Equivalence of several descriptions for $6 d$ SCFT, JHEP 01 (2017) 093 [arXiv:1607.07786] [INSPIRE].

[30] I. Brunner and A. Karch, Branes at orbifolds versus Hanany Witten in six-dimensions, JHEP 03 (1998) 003 [hep-th/9712143] [INSPIRE].

[31] A. Hanany and A. Zaffaroni, Branes and six-dimensional supersymmetric theories, Nucl. Phys. B 529 (1998) 180 [hep-th/9712145] [INSPIRE].

[32] A. Sen, F-theory and orientifolds, Nucl. Phys. B 475 (1996) 562 [hep-th/9605150] [INSPIRE]. 
[33] C. Hwang, J. Kim, S. Kim and J. Park, General instanton counting and 5d SCFT, JHEP 07 (2015) 063 [Addendum ibid. 04 (2016) 094] [arXiv: 1406.6793] [INSPIRE].

[34] H. Hayashi, S.-S. Kim, K. Lee and F. Yagi, Discrete theta angle from an O5-plane, JHEP 11 (2017) 041 [arXiv: 1707.07181] [INSPIRE].

[35] H. Hayashi, S.-S. Kim, K. Lee, M. Taki and F. Yagi, More on $5 d$ descriptions of $6 d$ SCFTs, JHEP 10 (2016) 126 [arXiv:1512.08239] [INSPIRE].

[36] H. Hayashi, S.-S. Kim, K. Lee and F. Yagi, 6d SCFTs, 5d dualities and Tao web diagrams, JHEP 05 (2019) 203 [arXiv:1509.03300] [INSPIRE].

[37] S. Cheng and S.-S. Kim, Refined topological vertex for $5 d \operatorname{Sp}(N)$ gauge theories with antisymmetric matter, arXiv:1809.00629 [INSPIRE].

[38] J. Kim, S. Kim and K. Lee, Higgsing towards E-strings, arXiv:1510.03128 [INSPIRE].

[39] M. Taki, Surface operator, bubbling Calabi-Yau and AGT relation, JHEP 07 (2011) 047 [arXiv: 1007.2524] [INSPIRE].

[40] S.N. Ruijsenaars, Hilbert-Schmidt operators vs. integrable systems of elliptic Calogero-Moser type IV. The relativistic heun (van Diejen) case, Symmetry, integrability and geometry: methods and applications, (2015) [arXiv:1404.4392].

[41] B. Haghighat, J. Kim, W. Yan and S.-T. Yau, D-type fiber-base duality, JHEP 09 (2018) 060 [arXiv: 1806.10335] [INSPIRE].

[42] J. Chen, B. Haghighat, H.-C. Kim and M. Sperling, Elliptic quantum curves of class $\mathcal{S}_{k}$, arXiv:2008.05155 [INSPIRE].

[43] T. Kimura and Y. Sugimoto, Quantum mirror curve of periodic chain geometry, JHEP 04 (2019) 147 [arXiv: 1810.01885] [inSPIRE].

[44] A. Iqbal and A.-K. Kashani-Poor, The vertex on a strip, Adv. Theor. Math. Phys. 10 (2006) 317 [hep-th/0410174] [INSPIRE].

[45] Y. Konishi and S. Minabe, Flop invariance of the topological vertex, Int. J. Math. 19 (2008) 27 [math.AG/0601352] [INSPIRE].

[46] M. Taki, Flop invariance of refined topological vertex and link homologies, arXiv:0805.0336 [INSPIRE].

[47] V. Mitev, E. Pomoni, M. Taki and F. Yagi, Fiber-base duality and global symmetry enhancement, JHEP 04 (2015) 052 [arXiv: 1411.2450] [INSPIRE]. 